Utilização de análise de componentes principais em séries temporais

Sérgio Coichev Teixeira

DissertaÇÃo APRESENTADA

$\mathrm{AO}$

Instituto DE Matemática e Estatística

DA

Universidade De SÃo Paulo

PARA

OBTENÇÃO DO TÍTULO

$\mathrm{DE}$

Mestre em CiÊnCIAS

Programa: Estatística

Orientador: Profa. Dra. Lúcia Pereira Barroso

São Paulo, Fevereiro de 2013 


\section{Utilização de análise de componentes principais em séries temporais}

Este exemplar corresponde à redação final da dissertação devidamente corrigida e defendida por Sérgio Coichev Teixeira e aprovada pela Comissão Julgadora.

Comissão Julgadora:

- Profa. Dra. Lúcia Pereira Barroso (orientadora) - IME-USP

- Profa. Dra. Clélia Maria de Castro Toloi - IME-USP

- Profa. Dra. Thelma Sáfadi - UFLA 


\section{Agradecimentos}

Gostaria de agradecer a todos, que diretamente ou indiretamente, contribuíram e tornaram possível a realização deste trabalho.

Agradeço especialmente minha orientadora Profa. Dra. Lúcia Pereira Barroso pela paciência, disponibilidade, confiança e pelas indispensáveis e incontáveis conversas necessárias para a construção deste trabalho.

Sou grato pelo apoio e prestatividade dos meus familiares que proporcionaram condições para o início desta jornada de estudo e por sempre estarem presentes e dispostos a me ajudar.

Agradeço também a minha esposa pelo suporte emocional, companhia, paciência e por entender minhas decisões e meus momentos de estresse.

Por fim, aos meus amigos que acreditaram que este trabalho poderia ser concluído com sucesso. 


\section{Resumo}

\section{(Utilização de análise de componentes principais em séries temporais)}

Um dos principais objetivos da análise de componentes principais consiste em reduzir o número de variáveis observadas em um conjunto de variáveis não correlacionadas, fornecendo ao pesquisador subsídios para entender a variabilidade e a estrutura de correlação dos dados observados com uma menor quantidade de variáveis não correlacionadas chamadas de componetes principais.

A técnica é muito simples e amplamente utilizada em diversos estudos de diferentes áreas. Para construção, medimos a relação linear entre as variáveis observadas pela matriz de covariância ou pela matriz de correlação.

Entretanto, as matrizes de covariância e de correlação podem deixar de capturar importante informações para dados correlacionados sequencialmente no tempo, autocorrelacionados, desperdiçando parte importante dos dados para interpretação das componentes.

Neste trabalho, estudamos a técnica de análise de componentes principais que torna possível a interpretação ou análise da estrutura de autocorrelação dos dados observados. Para isso, exploramos a técnica de análise de componentes principais para o domínio da frequência que fornece para dados autocorrelacionados um resultado mais específico e detalhado do que a técnica de componentes principais clássica. Pelos métodos SSA (Singular Spectrum Analysis) e MSSA (Multichannel Singular Spectrum Analysis), a análise de componentes principais é baseada na correlação no tempo e entre as diferentes variáveis observadas. Essas técnicas são muito utilizadas para dados atmosféricos na identificação de padrões, tais como tendência e periodicidade.

Palavras-chave:Análise de componentes principais, Análise de componentes principais para domínio da frequência, SSA, MSSA. 


\section{Abstract}

\section{(Use of principal component analysis in time series)}

The main objective of principal component analysis (PCA) is to reduce the number of variables in a small uncorrelated data sets, providing support and helping researcher understand the variation present in all the original variables with small uncorrelated amount of variables, called components. The principal components analysis is very simple and frequently used in several areas.

For its construction, the components are calculated through covariance matrix. However, the covariance matrix does not capture the autocorrelation information, wasting important information about data sets.

In this research, we present some techniques related to principal component analysis, considering autocorrelation information. However, we explore the principal component analysis in the domain frequency, providing more accurate and detailed results than classical component analysis time series case.

In subsequent method SSA (Singular Spectrum Analysis) and MSSA (Multichannel Singular Spectrum Analysis), we study the principal component analysis considering relationship between locations and time points. These techniques are broadly used for atmospheric data sets to identify important characteristics and patterns, such as tendency and periodicity.

Keywords: Principal Component Analysis, Principal Component Analysis in the Frequency Domain, SSA, MSSA 


\section{Sumário}

1 Introdução $\quad 1$

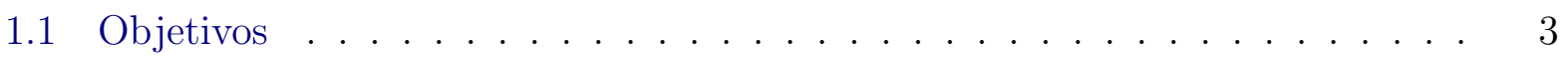

1.2 Contribuições . . . . . . . . . . . . . . . . . . . . . 3

1.3 Organização do Trabalho . . . . . . . . . . . . . . . . . . . 4

2 Análise de Componentes Principais (PCA) 5

2.1 Aplicação da Análise de Componentes Principais aos Dados fMRI . . . . . . 6

2.2 Análise e Resultados . . . . . . . . . . . . . . . . . . . . . . . 9

3 Análise Espectral e Domínio do Tempo $\times$ Frequência 11

3.1 Funções Periódicas . . . . . . . . . . . . . . . . . . . . . . . . 13

3.2 Estimador de Mínimos Quadrados com Frequência Conhecida . . . . . . . . 14

3.3 Estimador de Mínimos Quadrados com Frequência Desconhecida . . . . . . . 15

3.4 Análise Espectral . . . . . . . . . . . . . . . . . . . . . . 16

3.5 Transformada de Fourier Discreta . . . . . . . . . . . . . . . . 16

3.6 Densidade Espectral . . . . . . . . . . . . . . . . . . . 17

3.7 Periodograma . . . . . . . . . . . . . . . . . . . 19

4 Análise de Componentes Principais no Domínio da Frequência 21

4.1 Estimador para a Matriz de Densidade Espectral e Escolha do Parâmetro da Frequência $\lambda \ldots \ldots \ldots \ldots \ldots$. . . . . . . . . . . . . . . . . . . . . .

4.2 Aplicação da Análise de Componentes Principais no Domínio da Frequência aos Dados fMRI . . . . . . . . . . . . . . . . . . . . . . 24

4.3 Sobre os Dados e Resultados . . . . . . . . . . . . . . . . . . . . 28 
4.4 Método Clássico × Domínio da Frequência . . . . . . . . . . . . . . . . 28

5 SSA Singular Spectrum Analysis $\quad 31$

5.1 Primeira Etapa: Decomposição . . . . . . . . . . . . . . . . . . . . . 32

5.2 Segunda Etapa: Reconstrução . . . . . . . . . . . . . . . . . . . . . . . . 33

5.3 SSA e Análise de Fourier . . . . . . . . . . . . . . . . . . . . . . 36

5.4 Escolha do Tamanho da Janela $M \ldots$. . . . . . . . . . . . . . . . . . . . 37

5.4.1 Escolha do Tamanho da Janela para Extração de Tendência . . . . . 37

5.4.2 Escolha do Tamanho da Janela para Alisamento de Curva . . . . . . 38

5.4.3 Escolha do Tamanho da Janela para Séries Periódicas . . . . . . . . . 38

5.4.4 Escolha do Tamanho da Janela para Séries Desconhecidas . . . . . . 38

5.5 Quantidade de Grupos para Reconstrução das Séries . . . . . . . . . . . . . 39

5.6 Aplicação de SSA para Dados fMRI . . . . . . . . . . . . . . . . . . . . . . 39

6 MSSA Multichannel Singular Spectrum Analysis $\quad 45$

6.1 Escolha do Tamanho da Janela $M$ e da Quantidade de Grupos . . . . . . . . 46

6.2 Primeira Etapa: Decomposição . . . . . . . . . . . . . . . . . . . . . 46

6.3 Segunda Etapa: Reconstrução . . . . . . . . . . . . . . . . . . . . . . . . 50

6.4 Aplicação de MSSA para Dados fMRI . . . . . . . . . . . . . . . . . . . . 53

6.5 Sobre os Dados e Resultados . . . . . . . . . . . . . . . . . . . 59

7 Considerações Finais $\quad 63$

7.1 Conclusões . . . . . . . . . . . . . . . . . . . . . 63

7.2 Sugestões para Pesquisas Futuras . . . . . . . . . . . . . . . . . . 64

Referências Bibliográficas $\quad 65$ 


\section{Capítulo 1}

\section{Introdução}

Em muitos estudos multivariados, nos deparamos com a ausência de análises voltadas para estrutura temporal dos dados observados. Com isso, parte importante das informações são desperdiçadas podendo gerar impactos nos resultados e conclusões do estudo.

Neste trabalho, abordamos a técnica de análise de componentes principais para dados autocorrelacionados. Segundo Wilks [2011] a análise de componentes principais é possivelmente a técnica multivariada mais utilizada para dados atmosféricos e sem dúvidas é muito conhecida e utilizada em outras áreas.

A análise de componentes principais foi inicialmente descrita por Pearson [1901] e desenvolvida posteriormente por Hotelling [1933] e, ao longo dos anos e com avanços computacionais, a técnica tornou-se conhecida e amplamente utilizada em diversos estudos de diferentes áreas. Na análise, o objetivo principal é reduzir o número de variáveis observadas, $X_{1}, \ldots, X_{p}$, produzindo variáveis não correlacionadas, $Y_{1}, \ldots, Y_{p}$ que são combinações lineares das variáveis observadas e que representem o máximo possível da variabilidade de $X_{1}, \ldots, X_{p}$. Neste caso, as variáveis $Y^{\prime} s$ são chamadas de componentes principais.

O resultado será interessante quando parte representativa da variação dos dados observados estejam contidas em uma pequena quantidade de componentes, de forma a reduzir o volume de observações $X_{1}, \ldots, X_{p}$ em uma quantidade $Y_{1}, \ldots, Y_{d}, d<p$, de variáveis não correlacionadas. A análise de componentes principais não apresenta bons resultados quando as variáveis observadas são não correlacionadas.

Na grande maioria dos trabalhos publicados encontramos a técnica pela notação PCA (Prin- 
cipal Component Analysis), mas pode ser encontrada com outras notações. Em dados atmosféricos, a técnica foi introduzida por Obukhov [1947], e tornou-se popular com Lorenz [1956], que introduziu a notação EOF (Empirical Orthogonal Function). Por isso, em alguns trabalhos de dados atmosféricos, como em von Storch e Zwiers [2002], Cai e Baines [2001], Kim e Wu [1998], encontramos a notação EOF no lugar de PCA.

Assim como há diferenças na notação, encontramos muitas diferenças ou particularidades na natureza dos dados observados de um estudo para o outro.

Na prática, encontramos alguns estudos em que a estrutura temporal dos dados foi ignorada para a análise de PCA e para considerarmos essa estrutura, faremos a análise de componentes principais no domínio da frequência, que consiste em avaliar as componentes principais das séries segundo relações pertinentes da densidade espectral de cada uma das séries observadas. Veremos que a densidade espectral $f(\lambda)$, de uma série de tempo $X_{t}$, pode ser interpretada como a decomposição da variância em intervalo de frequência $\lambda$ entre $[-\pi, \pi]$. A principal vantagem em utilizarmos a análise de componentes principais no domínio da frequência consiste na possibilidade de gerar diferentes componentes para diferentes frequências, fornecendo um resultado mais elaborado e explicativo. Esta análise de componentes principais torna-se mais interessante quando as densidades espectrais das séries observadas são não idênticas ou quando as variâncias de cada uma das séries não sejam tão bem explicadas predominantemente por uma frequência $\lambda$.

Podemos ainda usar o método de componentes principais para séries de tempo para encontrar padrões como: tendência, oscilação, periodicidade e ruído. Este tipo de análise é conhecida como SSA (Singular Spectrum Analysis).

A ideia consiste em trabalhar com a estrutura de autocovariância de uma série $X_{t}$ com o objetivo de decompor a série observada em componentes aditivas que representam padrões da série.

Esta análise envolve a definição de um parâmetro $M$ chamado de tamanho de janela. O parâmetro define a quantidade de componentes geradas e tem grande importância na decomposição e identificação dos padrões da série $X_{t}$. Veremos que a escolha do parâmetro $M$ depende da complexidade da série. Assim, quanto mais informações tivermos sobre a série mais facilmente identificaremos o melhor valor $M$. 
No método SSA consideramos uma única série de tempo $X_{t}$. Na versão multivariada do método, conhecido como MSSA (Multichannel Singular Spectrum Analysis), estaremos interessados em capturar estruturas que considerem um comportamento mais abrangente, levando em consideração os efeitos entre um conjunto de $L$ séries de tempo.

Mostramos que PCA é um caso particular do MSSA, quando o tamanho da janela $M=1 \mathrm{e}$ a quantidade de séries $L>1$; por outro lado, quando $L=1$ e $M>1$ teremos o SSA.

Como no SSA, necessitamos definir a janela $M$ com a mesma finalidade descrita, ou seja, quanto mais informação sobre a série melhor será a escolha do parâmetro $M$.

A análise MSSA colabora de forma mais representativa que o método SSA aplicado para cada uma das $L$ séries, uma vez que MSSA identifica padrões ou estruturas globais de um fenômeno ou ocorrências, levando em consideração a autocorrelação de cada e entre cada uma das $L$ séries.

Ambos métodos, SSA e MSSA, têm ampla aplicação em estudos atmosféricos com o objetivo de capturar estruturas como efeitos sazonais ou periódicos.

\subsection{Objetivos}

O principal objetivo deste trabalho consiste em apresentar as técnicas: análise de componentes principais (PCA), análise de componentes principais para domínio da frequência, SSA e MSSA, de forma a compará-las, descrevendo particularidades e ilustrando aplicações por meio de exemplos de cada uma das técnicas.

\subsection{Contribuições}

Com a descrição de cada uma das técnicas, contribuímos mostrando que a análise de componetes principais para séries de tempo pode ser aplicada de outras formas, que não a usual.

Neste trabalho abordamos a técnica de análise de componentes principais para o domínio da frequência e mostramos que podemos enriquecer a análise e extrair informações pertinentes se levarmos em consideração as informações da densidade espectral das séries. 
As análises SSA e MSSA são apresentadas como formas de utilizar a análise de componentes para observações autocorrelacionadas, com a finalidade de identificar padrões e estruturas de uma série no caso SSA e de uma forma global para $L$ séries no caso MSSA.

Encontramos grande parte dos trabalhos de SSA e MSSA em análise de dados atmosféricos. Com isso, desejamos contribuir deixando a técnica transparente e disponível para o uso em outras áreas ou natureza de dados.

\subsection{Organização do Trabalho}

No Capítulo 2, apresentamos a análise de componentes principais, caso clássico (PCA), com as principais propriedades e método de cálculo.

No Capítulo 3, antes de apresentarmos a análise de componentes principais para o domínio da frequência, descrevemos alguns conceitos relacionados à análise espectral, como densidade espectral e periodograma.

No Capítulo 4, descrevemos a análise de componentes principais para o domínio da frequência, em que destacamos as principais particularidades e similaridade do método com a análise de componentes principais (PCA).

Nos Capítulos 5 e 6, respectivamente, apresentamos as técnicas SSA e MSSA e no Capítulo 7 destacamos algumas sugestões para novos trabalhos e as principais conclusões obtidas neste trabalho. 


\section{Capítulo 2}

\section{Análise de Componentes Principais}

\section{(PCA)}

A técnica de análise de componentes principais foi inicialmente descrita por Pearson [1901] e posteriormente, por meios de métodos computacionais, aprimorada por Hotelling [1933]. Com o passar dos anos e evoluções tecnológicas e melhores computadores, o método tornou-se mais utilizado.

A análise de componentes principais é simples, o objetivo da análise é baseado no fato de que partindo de um conjunto de variáveis $\mathbf{X}=\left(X_{1}, \ldots, X_{p}\right)^{\prime}$ é possível encontrar combinações destas para produzir índices $\mathbf{Y}=\left(Y_{1}, \ldots, Y_{p}\right)^{\prime}$, não correlacionados, na ordem da sua importância e que descrevam as variações dos dados, tal que a maior parte da variação seja representada adequadamente por poucas variáveis do vetor Y. Com isso, desejamos encontrar a combinação linear, tal que:

$$
\mathbf{Y}=\mathbf{c}^{\prime} \mathbf{X}=c_{1} X_{1}+\ldots+c_{p} X_{p}
$$

em que c é um vetor fixado. Assim, procuramos a combinação de forma que $\operatorname{Var}(Y)$ seja a maior possível, restrito a $\mathbf{c}^{\prime} \mathbf{c}=1$. Podemos escrever $\operatorname{Var}(Y)=\mathbf{c}^{\prime} \boldsymbol{\Sigma}_{\mathbf{x}} \mathbf{c}$ em que $\boldsymbol{\Sigma}_{\mathbf{x}}$ é $(p \times p)$ matriz de covariância de $\mathbf{X}=\left(X_{1}, \ldots, X_{p}\right)^{\prime}$. A forma de solucionar o problema é encontrar c tal que:

$$
\max _{\mathbf{c} \neq 0} \frac{\mathbf{c}^{\prime} \Sigma_{\mathbf{x}} \mathbf{c}}{\mathbf{c}^{\prime} \mathbf{c}}, \mathbf{c}^{\prime} \mathbf{c}=1
$$


Denotamos respectivamente os autovalores e autovetores de $\boldsymbol{\Sigma}_{\mathbf{x}}$ pelos pares $\left(\lambda_{1}, \mathbf{e}_{\mathbf{1}}\right), \ldots$, $\left(\lambda_{p}, \mathbf{e}_{\mathbf{p}}\right)$, em que os autovalores são tais que: $\lambda_{1} \geq \lambda_{2} \geq \ldots \geq \lambda_{p} \geq 0$ e os autovetores, $\mathbf{e}_{\mathbf{1}}, \ldots, \mathbf{e}_{\mathbf{p}}$ são de tamanho unitário. A solução para $\max _{\mathbf{c} \neq 0} \frac{\mathbf{c}^{\prime} \boldsymbol{\Sigma}_{\mathbf{x}} \mathbf{c}}{\mathbf{c}^{\prime} \mathbf{c}}$ é escolher $\mathbf{c}=\mathbf{e}_{\mathbf{1}}$, para o caso em que a combinação linear $Y_{1}=\mathbf{e}_{\mathbf{1}}^{\prime} \mathbf{X}$ tenha máxima variância e $\operatorname{Var}\left(Y_{1}\right)=\lambda_{1}$. Em outras palavras:

$$
\max _{\mathbf{c} \neq 0} \frac{\mathbf{c}^{\prime} \boldsymbol{\Sigma}_{\mathbf{x}} \mathbf{c}}{\mathbf{c}^{\prime} \mathbf{c}}=\frac{\mathbf{e}_{\mathbf{1}}^{\prime} \Sigma_{\mathbf{x}} \mathbf{e}_{\mathbf{1}}}{\mathbf{e}_{\mathbf{1}}^{\prime} \mathbf{e}_{\mathbf{1}}}=\lambda_{1} .
$$

A combinação linear $Y_{1}=\mathbf{e}_{1}^{\prime} \mathbf{X}$ é chamada de primeira componente principal; a segunda componente é definida pela combinação linear $Y_{2}=\mathbf{c}^{\prime} \mathbf{X}$ que maximiza $\operatorname{Var}\left(Y_{2}\right)$ sujeito a $\mathbf{c}^{\prime} \mathbf{c}=1$, tal que $\operatorname{cov}\left(Y_{1}, Y_{2}\right)=0$. Assim a solução é escolher $\mathbf{c}=\mathbf{e}_{\mathbf{2}}$, ou seja, $Y_{2}=\mathbf{e}_{\mathbf{2}}^{\prime} \mathbf{X}$ e $\operatorname{Var}\left(Y_{2}\right)=\lambda_{2}$ e de forma similar é possível encontrar todas as componentes principais para todo $\lambda_{j}, j=1,2, \ldots p$. Desta forma, teremos como resultado: $Y_{1}, \ldots, Y_{p}$ como as componentes principais e autovalores $\lambda_{1}, \ldots, \lambda_{p}$ como as variâncias das componentes. Assim, o total da variância será dado por $\sum_{i=1}^{p} \lambda_{i}$, e a quantidade ou parte da variância associada à $j$-ésima componente é $\frac{\lambda_{j}}{\sum_{j=1}^{p} \lambda_{j}}$.

Mais detalhes sobre o método podem ser encontrados em Johnson e Wichern [1998] e Rencher [1997].

\subsection{Aplicação da Análise de Componentes Principais aos Dados fMRI}

Neste trabalho, escolhemos analisar os dados relativos a fMRI (functional Magnetic Resonance Imaging) disponíveis em Shumway e Stoffer [2006]. Embora a análise desses dados já tenha sido apresentada nesse texto, fazemos a análise com outras técnicas para podermos compará-las.

De forma simplificada fMRI é um procedimento utilizado para relacionar atividades cerebrais a alterações ou mudanças associadas ao fluxo sanguíneo.

Neste exemplo, foram medidos níveis de oxigenação do sangue após a aplicação de estímulos em algumas regiões do cérebro. Os estímulos foram aplicados em 4 regiões do cortex, $X_{1 t}, X_{2 t}, X_{3 t}, X_{4 t}$, em 2 regiões do tálamo, $X_{5 t}, X_{6 t}$ e em duas regiões do cerebelo $X_{7 t}, X_{8 t}$. 
Desta forma, $\mathbf{X}_{t}=\left(X_{1 t}, X_{2 t}, X_{3 t}, X_{4 t}, X_{5 t}, X_{6 t}, X_{7 t}, X_{8 t}\right)^{\prime}$ representa medidas do nível de oxigenação do sangue observadas na t-ésima unidade de tempo.

Os estímulos aconteceram a cada 32 segundos e então interrompidos por mais 32 segundos, assim o período do sinal é de 64 segundos. Contudo, a amostra é efetuada retirando uma observação a cada dois segundos dos 256 segundos do estudo. Temos para análise 128 observações para cada uma das 8 séries $\mathbf{X}_{t}=\left(X_{1 t}, X_{2 t}, X_{3 t}, X_{4 t}, X_{5 t}, X_{6 t}, X_{7 t}, X_{8 t}\right)^{\prime}$.

Apresentamos na Figura 2.1, os valores do nível de oxigenação para as 8 variáveis. Notamos, na figura, que regiões diferentes do cérebro respondem de formas diferentes ao estímulo, tal como se observa na sazonalidade do nível de oxigenação para a região do cortex e que não foram observadas, por exemplo, para as regiões do cerebelo.

A matriz de covariância $\boldsymbol{\Sigma}_{\mathbf{x}}$ é desconhecida e é estimada da forma usual por meio de $\mathbf{S}_{\mathbf{x}}$. Para encontrarmos as componetes $Y_{1}, \ldots, Y_{8}$, precisamos encontrar a solução para:

$$
\max _{\mathbf{c} \neq 0} \frac{\mathbf{c}^{\prime} \mathbf{S}_{\mathbf{x}} \mathbf{c}}{\mathbf{c}^{\prime} \mathbf{c}}, \mathbf{c}^{\prime} \mathbf{c}=1
$$

A estimativa dos autovalores foi $(4,34 ; 1,13 ; 0,79 ; 0,59 ; 0,42 ; 0,35 ; 0,22 ; 0,11)$ e portanto, temos para os dados observados que a primeira componente $Y_{1}$ explica 54,4\% da variação dos dados e, respectivamente para as demais componentes, as seguintes contribuições: (14,2\%; $9,9 \% ; 7,5 \% ; 5,3 \% ; 4,5 \% ; 2,8 \% ; 1,4 \%)$.

Analisando a primeira componente, temos o autovetor associado $\mathbf{e}_{\mathbf{1}}$, com cargas associadas respectivamente às variáveis observadas $X_{1 t}, . ., X_{8 t}$ respectivamente: $(0,45 ; 0,40 ; 0,42 ; 0,32$; 0,38; -0,08; 0,22; 0,39). Assim, a primeira componente explica $54,4 \%$ da variabilidade e pelas cargas encontradas parece descrever o efeito periódico do nível de oxigenação do sangue após o estímulo. Como pode-se observar na Figura 2.1, as séries que representam o nível de oxigenação após o estímulo aplicado na região 2 do tálamo e na região 1 do cerebelo parecem diferentes das demais e isso está refletido nas suas cargas na primeira componente, que são as menores.

Na Figura 2.2, temos a primeira componente que, conforme havíamos descrito, evidencia a periodicidade esperada. 

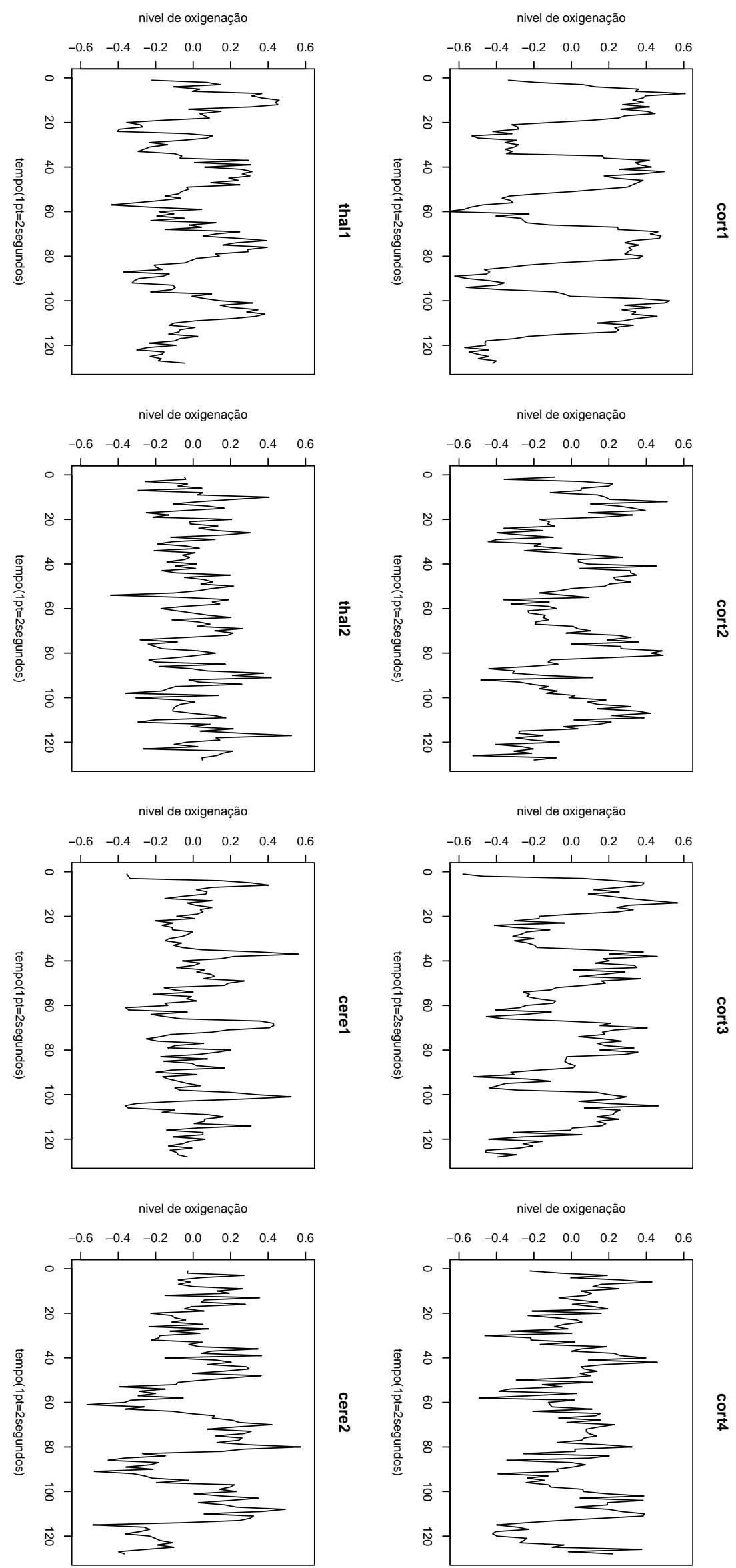

Figura 2.1: Nivel de oxigenação do sangue para as regiões: $X_{1 t}=$ cort1, $X_{2 t}=$ cort2, $X_{3 t}=$ cort3, $X_{4 t}=$ cort $4, X_{5 t}=$ thal1,$X_{6 t}=$ thal2, $X_{7 t}=$ cere $1, X_{8 t}=$ cere2 


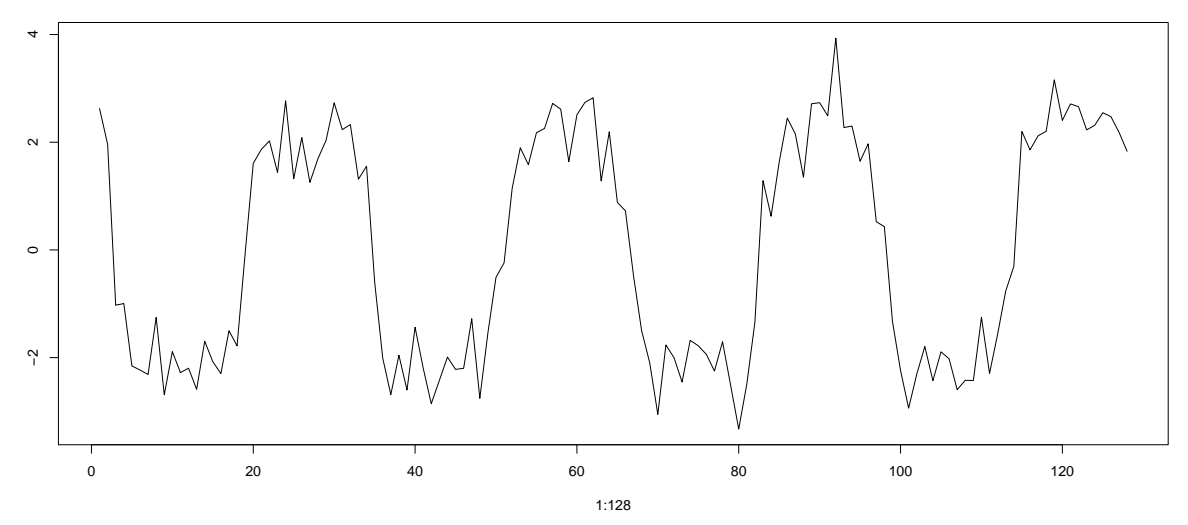

Figura 2.2: Primeira componente principal para dados fMRI

As cargas associadas à segunda componente principal $(0,00 ; 0,10 ;-0,13 ;-0,05 ; 0,18 ;-0,76$; $-0,59 ; 0,07)$ são maiores para as regiões 2 do tálamo e 1 do cerebelo. As duas primeiras componentes explicam $68,6 \%$ da variância total dos dados.

\subsection{Análise e Resultados}

Os dados de fMRI apresentam uma estrutura temporal periódica dada pela resposta ao estímulo aplicado a cada 32 segundos. Contudo, nesta análise, aplicamos a técnica diretamente aos dados não considerando a estrutura temporal das observações.

A seguir, descrevemos a análise de componentes principais para o domínio da frequência, no qual avaliamos o mesmo exemplo levando em consideração a estrutura temporal e periódica dos dados. Para isso, precisamos transformar os dados observados para o domínio da frequência, ou seja, transformamos as séries de tempo em quantidades de sinal por faixas de frequência. Mostramos que o resultado será mais abrangente, possibilitando a análise de componentes para cada faixa de frequência desejada. Contudo, antes de descrevermos o método precisamos descrever alguns conceitos sobre domínio da frequência e análise espectral. 
10 ANÁLISE DE COMPONENTES PRINCIPAIS (PCA) 


\section{Capítulo 3}

\section{Análise Espectral e Domínio do}

\section{Tempo $\times$ Frequência}

Iniciamos este capítulo descrevendo alguns conceitos sobre domínio do tempo e da frequência e em seguida descrevemos alguns conceitos sobre análise espectral, tais como: função de densidade espectral e estimadores do espectro. Neste trabalho apresentamos um resumo desses conceitos, mas mais detalhes podem ser encontrados em Morettin e Toloi [2006] e Morettin [1999].

\section{Domínio do Tempo}

O termo domínio do tempo é usado para descrever a análise de funções que assumem valores com relação ao tempo, seja em instantes separados de tempo para o caso discreto ou conhecido a cada e todo instante de tempo no caso contínuo.

O enfoque sobre a análise do domínio do tempo está voltada para modelos paramétricos (com número finito de parâmetros), como por exemplo modelos ARIMA, enquanto na análise do domíno da frequência os modelos propostos são não paramétricos.

\section{Domínio da Frequência}

O domínio da frequência pressupõe que fenômenos no domínio do tempo se repetem em in- 
tervalos iguais a $T$, sendo $T$ o período de tempo que contém um ciclo do sinal de frequência $\lambda$. Assim, temos a relação $\lambda=1 / T$.

O termo domínio da frequência é usado para descrever a análise de funções que assumem valores com respeito à frequência. Desta forma, no domínio da frequência podemos analisar variáveis pela quantidade de sinal por unidade de frequência.

De forma simplificada um gráfico no domínio do tempo mostra como um sinal varia ao longo do tempo, já um gráfico no domínio da frequência mostra quanto do sinal reside em cada faixa de frequência.

\section{Uso do Domínio do Tempo e Frequência}

Uma série no domínio do tempo pode ser transformada para o domínio da frequência por meio de uma transformação dos dados como a transformada de Fourier, que decompõe a série segundo as suas componentes de intensidade.

A escolha do tipo do domínio está relacionada ao objetivo do estudo, geralmente o uso pelo domínio da frequência envolve análise de sinais/ondas principalmente nos campos de Telecomunicações, Meteorologia e Oceanografia.

\section{Análise de Fourier}

A análise de Fourier é geralmente utilizada para resolver equações diferenciais parciais, como por exemplo, na aplicação nas equações de onda e calor.

A ideia básica é aproximar uma função $g(t)$, função do tempo, por uma combinação linear de componentes senoidais, em que os coeficientes são resultados da transformada de Fourier da série.

A seguir, mostramos algumas definições sobre funções periódicas as quais terão papel fundamental na estimação dos parâmetros na análise de Fourier. 


\subsection{Funções Periódicas}

Dizemos que uma função $g(t), t \in \mathbb{R}$ é periódica de período $p$ se

$$
g(t)=g(t+k p), k=0, \pm 1, \pm 2, \ldots
$$

Supondo $p \neq 0$, dizemos que $g(t)$ é um harmônico da frequência angular $\lambda$ e amplitude $R$, se

$$
g(t)=R \cos (\lambda t+\phi)
$$

Temos então um harmônico com frequência angular $\lambda$ e período $p=\frac{2 \pi}{\lambda}$, a frequência angular $\lambda$ fornece o número de ciclos completos em $2 \pi$ unidades de tempo e $\phi$ é chamado de fase e fornece o deslocamento da onda em relação à origem. Desta forma, podemos considerar a frequência em ciclos por unidade de tempo, $\nu$, de tal forma que $\lambda=2 \pi \nu$ e segue também que $p=\frac{1}{\nu}$.

Assim, por exemplo, uma onda com período $p=2$ segundos tem frequência $\nu=\frac{1}{2}$ ciclo por segundo e frequência angular $\lambda=2 \pi \frac{1}{2}=\pi$ radianos por segundo. Resumidamente dizer que $\nu=0,5$ significa que em um segundo ocorre 0,5 de um ciclo.

Utilizando identidade trigonométrica podemos escrever $g(t)$ da seguinte forma:

$$
g(t)=A \cos (\lambda t)+B \operatorname{sen}(\lambda t), A=R \cos (\phi) \text { e } B=-R \operatorname{sen}(\phi) .
$$

Sob suposições diversas sobre seu comportamento, podemos escrever $g(t), t \in \mathbb{R}$ como uma mistura de séries com múltiplas frequências e amplitudes.

$$
g(t)=\sum_{\lambda}[A \cos (\lambda t)+B \operatorname{sen}(\lambda t)]
$$

A somatória pode ser uma soma infinita ou finita ou mesmo uma integral. Dizemos ainda que a somatória acima é uma representação espectral de $g(t)$.

Em aplicações como metereologia e oceonografia, estamos buscando a periodicidade dos dados observados, por isso duas situações acontecem: 
i) conhecemos a frequência e queremos estimar amplitude e fase, como nos casos das marés onde as frequências são determinadas por instrumentos;

ii) queremos estimar amplitudes, frequência e fases, caso mais geral encontrado na prática.

\subsection{Estimador de Mínimos Quadrados com Frequência}

\section{Conhecida}

Suponha uma série temporal, $X_{1}, \ldots, X_{N}$, em que $X_{t}$ é representado por:

$$
X_{t}=\mu+R \cos (\lambda t+\phi)+\varepsilon_{t}
$$

ou, equivalentemente,

$$
X_{t}=\mu+A \cos (\lambda t)+B \operatorname{sen}(\lambda t)+\varepsilon_{t}
$$

em que $A=R \cos (\phi)$ e $B=-R \operatorname{sen}(\phi)$, com $\phi=$ ângulo de fase, $R=$ amplitude, $\lambda=$ frequência e $\varepsilon_{t}=$ componente aleatória.

Os estimadores de mínimos quadrados para $(\hat{\mu}, \hat{A}, \hat{B})$ serão obtidos quando minimizarmos a soma dos quadrados dos resíduos.

$$
S Q R(\mu, a, b)=\sum_{t=1}^{N}\left[\left(X_{t}-\mu-A \cos (\lambda t)-B \operatorname{sen}(\lambda t)\right]^{2}\right.
$$

e a solução será

$$
\begin{gathered}
\hat{\mu}=\frac{1}{N} \sum_{t=1}^{N} X_{t}=\bar{X} \\
\hat{A}=\frac{2}{N} \sum_{t=1}^{N} X_{t} \cos (\lambda t), \text { para } \lambda \neq \pi \\
\hat{B}=\frac{2}{N} \sum_{t=1}^{N} X_{t} \operatorname{sen}(\lambda t), \operatorname{para} \lambda \neq \pi \text { e } \hat{B}=0 \text { se } \lambda=\pi \\
\hat{R}^{2}=\hat{A}^{2}+\hat{B}^{2} \text { e } \hat{\phi}=\arctan \left(-\frac{\hat{B}}{\hat{A}}\right) .
\end{gathered}
$$


É possível encontrar uma solução aproximada, quando $\lambda \neq \frac{2 \pi k}{N}$ e não muito próxima de zero, dada por

$$
\begin{gathered}
\tilde{\mu}=\hat{\mu}=\bar{X} \\
\tilde{A}=\frac{2}{N} \sum_{t=1}^{N}\left(X_{t}-\bar{X}\right) \cos (\lambda t) \\
\tilde{B}=\frac{2}{N} \sum_{t=1}^{N}\left(X_{t}-\bar{X}\right) \operatorname{sen}(\lambda t) \\
\tilde{R}=\tilde{A}^{2}+\tilde{B}^{2} \text { e } \tilde{\phi}=\arctan \left(-\frac{\tilde{B}}{\tilde{A}}\right) .
\end{gathered}
$$

\subsection{Estimador de Mínimos Quadrados com Frequência Desconhecida}

A ideia é similar ao caso com frequência conhecida, contudo precisamos incluir a estimação da frequência $\lambda$.

Nesta seção os estimadores para $\mu, A, B$ dependerão de $\lambda$ e serão denotados por $\hat{\mu}(\lambda), \tilde{A}(\lambda)$ e $\tilde{B}(\lambda)$, respectivamente.

O melhor valor de $\lambda$ é o valor que minimiza a soma dos quadrados residuais ou, equivalentemente, maximiza a quantidade:

$$
\tilde{R}(\lambda)^{2}=\tilde{A}(\lambda)^{2}+\tilde{B}(\lambda)^{2}
$$

$\operatorname{com} \tilde{A}(\lambda)$ e $\tilde{B}(\lambda)$ dados por

$$
\begin{aligned}
& \tilde{A}(\lambda)=\frac{2}{N} \sum_{t=1}^{N}\left(X_{t}-\bar{X}\right) \cos (\lambda t) \\
& \tilde{B}(\lambda)=\frac{2}{N} \sum_{t=1}^{N}\left(X_{t}-\bar{X}\right) \operatorname{sen}(\lambda t),
\end{aligned}
$$

lembrando que $\tilde{A}$ e $\tilde{B}$ são soluções aproximadas dos estimadores de mínimos quadrados 
quando $\lambda \neq \frac{2 \pi k}{N}, k=1,2, \ldots, \frac{N}{2}$.

Maximizar $\tilde{R}(\lambda)^{2}$ é equivalente a maximizar a quantidade:

$$
I(\lambda)=\frac{N}{8 \pi} \tilde{R}^{2}(\lambda)=\frac{1}{2 \pi N}\left[\left(\sum_{t=1}^{N}\left(X_{t}-\bar{X}\right) \cos (\lambda t)\right)^{2}+\left(\sum_{t=1}^{N}\left(X_{t}-\bar{X}\right) \operatorname{sen}(\lambda t)\right)^{2}\right]
$$

em que $\mathrm{I}(\lambda)$ é denominado periodograma.

Assim, estimamos $\lambda$ maximizando $\mathrm{I}(\lambda)$ e obtemos os demais estimadores do modelo utilizando os estimadores para frequência conhecida.

\subsection{Análise Espectral}

A análise espectral para séries de tempo estacionárias $X_{t}$ decompõe a série em componentes senoidais com coeficientes aleatórios não correlacionados, juntamente com a correspondente decomposição em senoides da função de autocovariância $\gamma(\mathrm{t})$.

As áreas com interesse em procura de periodicidade dos dados, como metereologia e oceonografia são as mais interessadas neste tipo de análise.

\subsection{Transformada de Fourier Discreta}

Suponha $X_{t}, t=0,1, \ldots, N$ uma realização de um processo estacionário com média zero. Então

$$
d^{(N)}(\lambda)=\frac{1}{\sqrt{2 \pi N}} \sum_{t=1}^{N} X_{t} \exp (-i \lambda t),-\infty<\lambda<\infty,
$$

é denominada transformada de Fourier finita(TFF) de $\left(X_{1}, X_{2}, \ldots, X_{N}\right)$.

Embora, TFF seja definida para toda frequência, na prática a frequência é calculada da forma $\lambda_{j}=\frac{2 \pi j}{N}$, para $-\frac{N-1}{2} \leq j \leq \frac{N}{2}$. Assim, obtemos a transformada de Fourier discreta

$$
d_{j}^{(N)}(\lambda)=\frac{1}{\sqrt{2 \pi N}} \sum_{t=1}^{N} X_{t} \exp (-i 2 \pi j t / N), j=0,1, \ldots, \frac{N}{2} .
$$


Note que $d_{j}^{(N)}$ tem período $2 \pi$, portanto basta considerar $\lambda_{j}$ no intervalo $[-\pi, \pi]$.

Assumindo, pela definição, que $X_{t}$ tem média zero,

$$
E\left[d_{j}^{(N)}\right]=0
$$

e desta forma a variância é determinada por

$$
\operatorname{Var}\left[d_{j}^{(N)}\right]=E\left[d_{j}^{(N)}\right]^{2}
$$

É possível demonstrar que para $N$ grande, e se $f(\lambda)$ é contínua, podemos aproximar $E\left[d_{j}^{(N)}\right]^{2}$ por $f\left(\lambda_{j}\right)$, em que $f\left(\lambda_{j}\right)$ é a densidade espectral e será definida em seguida. Quanto mais suave for $f(\lambda)$ na vizinhança de $\lambda_{j}=\frac{2 \pi j}{N},-\frac{N-1}{2} \leq j \leq \frac{N}{2}$, melhor será a aproximação.

\subsection{Densidade Espectral}

Considere $X_{t}, \mathrm{t} \in \mathbb{Z}$, um processo estocástico estacionário com média zero e $\gamma(\tau)$, para $\tau=1, \ldots, \infty$, a sua matriz de autocovariância satisfazendo, como consequência de um processo estocástico estacionário, a condição de independência assintótica no sentido que valores bem separados no tempo sejam pouco dependentes, e que

$$
\sum_{\tau=1}^{\infty}|\gamma(\tau)|<\infty
$$

Como exemplo, consideramos o caso de um ruído branco com média 0, e variância $\sigma^{2}$. Desta forma temos

$$
\begin{gathered}
\gamma(\tau)=\sigma^{2}, \text { para } \tau=0 \\
\gamma(\tau)=0, \text { para }|\tau| \geq 1
\end{gathered}
$$

Assim, podemos definir a função densidade espectral ou espectro de $X_{t}$ da seguinte forma:

$$
f(\lambda)=\frac{1}{2 \pi} \sum_{\tau=-\infty}^{\infty} \gamma(\tau) \exp (-i \lambda \tau),-\infty<\lambda<\infty
$$


Com isso, $f(\lambda)$ é a transformada de Fourier de $\gamma(\mathrm{t})$, em que $\exp (i \lambda)=\cos (\lambda)+i \operatorname{sen}(\lambda)$, $i=\sqrt{-1}$, pela fórmula de Euler.

O espectro $f(\lambda)$ é limitado, não negativo, par e periódico de período $2 \pi$.

Como $f(\lambda)$ é periódico de período $2 \pi$, basta considerar o intervalo, $[-\pi, \pi]$ e ainda, como $f(\lambda)$ é par basta representá-lo de $[0, \pi]$ e com isso temos

$$
\gamma(\tau)=\int_{-\pi}^{\pi} f(\lambda) \exp (i \lambda \tau) d \lambda, \tau \in \mathbb{Z}
$$

ou seja, $\gamma(\tau)$ pode ser recuperada de $f(\lambda)$ utilizando a transformada inversa de Fourier.

Do ponto de vista da quantidade de informação, o espectro e a função de autocovariância são equivalentes.

O espectro $f(\lambda)$ pode ser interpretado como uma decomposição da variância do processo, de forma que o termo $f(\lambda) d \lambda$ é a contribuição da variância atribuída à componente do processo com frequência no intervalo $(\lambda, \lambda+d \lambda)$.

Desta forma, um pico no espectro indica uma contribuição importante à variância do processo. O gráfico $\lambda \times f(\lambda)$ pode ser pensado como uma análise de variância em que a coluna "efeito"é constituída pelas frequências.

Como exemplo, novamente consideramos o caso de um ruído branco com média 0 e variância $\sigma^{2}$ e desta forma temos:

$$
\begin{gathered}
\gamma(\tau)=\sigma^{2}, \text { para } \tau=0 \\
\gamma(\tau)=0, \text { para }|\tau| \geq 1 .
\end{gathered}
$$

Neste caso, temos $f(\lambda)=\frac{1}{2 \pi} \gamma(0)=\frac{\sigma^{2}}{2 \pi}$, para $-\pi \leq \lambda \leq \pi$.

Consideramos agora, como exemplo, um processo estacionário com frequência fixa $\lambda_{0}$.

$$
X_{t}=U_{1} \cos \left(2 \pi \lambda_{0} t\right)+U_{2} \operatorname{sen}\left(2 \pi \lambda_{0} t\right)
$$

em que $U_{1}$ e $U_{2}$ são independentes com média zero e variância $\sigma^{2}$. O número necessário para o processo completar um ciclo é exatamente $\frac{1}{\lambda_{0}}$. 
Desta forma :

$$
\gamma(h)=\sigma^{2} \cos \left(2 \pi \lambda_{0} h\right)=\int_{\frac{-1}{2}}^{\frac{1}{2}} \exp (i \lambda h 2 \pi) d F(\lambda) .
$$

Usando a integral de Riemann-Stieltjes, temos:

$$
\begin{gathered}
F(\lambda)=0, \text { para } \lambda<-\lambda_{0} \\
F(\lambda)=\frac{\sigma^{2}}{2}, \text { para }-\lambda_{0} \leq \lambda<\lambda_{0} \\
F(\lambda)=\sigma^{2}, \text { para } \lambda \geq \lambda_{0} .
\end{gathered}
$$

A função $F(\lambda)$ tem comportamento similar à função distribuição de probabilidade discreta, exceto que $F(\infty)=\sigma^{2}$ no lugar de 1 .

De fato $F(\lambda)$ é uma função de distribuição acumulada, não de probabilidade mas de variância associada com a frequência $\lambda_{0}$.

\subsection{Periodograma}

A aproximação de $E\left[d_{j}^{(N)}\right]^{2}$ por $f\left(\lambda_{j}\right)$ sugere que dada uma realização de $X_{t}, t=1, \ldots, N$, processo estacionário, obtemos como estimador para $f\left(\lambda_{j}\right)$ :

$$
I_{j}^{(N)}=\left|d_{j}^{(N)}\right|^{2}=\frac{1}{2 \pi N}\left|\sum_{t=1}^{N} X_{t} \exp \left(-i \lambda_{j} t\right)\right|^{2},
$$

ou, equivalentemente,

$$
I_{j}^{(N)}=\frac{1}{2 \pi} \sum_{h=-[N-1]}^{[N-1]} \hat{\gamma}(h) \exp \left(-i h \lambda_{j}\right)
$$

em que $I_{j}^{(N)}$ é denominado periodograma e pode ser também definido para qualquer frequência $\lambda \in[-\pi, \pi]$ e $\hat{\gamma}(h)=\frac{1}{N} \sum_{t=1}^{N-|h|} X_{t} X_{t+|h|}$, quando $E\left(X_{t}\right)=0$. Contudo, na prática, será calculado para um número finito de frequências. 


\section{Capítulo 4}

\section{Análise de Componentes Principais}

\section{no Domínio da Frequência}

A seguir, descrevemos a análise de componentes principais no domínio da frequência. Na análise avaliamos informações e relações pertinentes da densidade espectral de cada e entre cada uma das séries de tempo observadas. Mais detalhes sobre o método pode ser encontrado em Jolliffe [2002], Shumway e Stoffer [2006], Brillinger [2001], Lagerlund et al. [2004] e McDougall et al. [1995].

Para a análise do domínio da frequência, trabalhamos com valores complexos, desta forma, um vetor de séries de tempo $\mathbf{X}_{t}$ pode ser representado por $\mathbf{X}_{t}=\mathbf{X}_{1 t^{-}} i \mathbf{X}_{2 t}$, em que $\mathbf{X}_{1 t}$ é a parte real e $\mathbf{X}_{2 t}$ é a parte imaginária de $\mathbf{X}_{t}$. Para a técnica, trabalhamos com séries estacionárias e com média zero.

No caso de séries de tempo complexas, $\mathbf{X}_{t}=\mathbf{X}_{1 t}-i \mathbf{X}_{2 t}$, dizemos que o processo será estacionário se $\mathrm{E}\left(\mathbf{X}_{t}\right)$ e $\mathrm{E}\left(\mathbf{X}_{t} \mathbf{X}_{t}^{*}\right)$ existirem e forem independentes do tempo $t$, em que $\mathbf{X}_{t}^{*}$ é o transposto do complexo conjugado de $\mathbf{X}_{t}$.

Supondo que $\mathbf{X}_{t}$ tenha dimensão $(p \times 1)$, então a função de autocovariância terá dimensão $(p \times p)$, e será dada por

$$
\boldsymbol{\Gamma}_{\mathbf{x} \mathbf{x}}(h)=E\left(\mathbf{X}_{t+h} \mathbf{X}_{t}^{*}\right)-E\left(\mathbf{X}_{t+h}\right) E\left(\mathbf{X}_{t}^{*}\right) .
$$


Para facilitar a notação, chamamos $\boldsymbol{\Gamma}_{\mathbf{x x}}(h)=\left[\gamma_{i j}(h)\right]$, para $i, j=1, \ldots, p$. Seguem abaixo, algumas propriedades de $\boldsymbol{\Gamma}_{\mathbf{x x}}(h)$ :

$$
\begin{gathered}
(i) \gamma_{i i}(0) \geq 0 \\
(i i)\left|\gamma_{i j}(h)\right|^{2} \leq \gamma_{i i}(0) \gamma_{j j}(0) \text { para todo } h \\
(i i i) \boldsymbol{\Gamma}_{\mathbf{x x}}(h)=\boldsymbol{\Gamma}_{\mathbf{x x}}(h)^{*} .
\end{gathered}
$$

Para um processo estacionário com média zero com $\mathbf{X}_{t}=\left(X_{1 t}, \ldots, X_{p t}\right)^{\prime}$ de dimensão $(p \times 1)$ e supondo $\boldsymbol{\Gamma}_{\mathbf{x x}}(h)$ não negativa definida e $\sum_{h}\left|\boldsymbol{\Gamma}_{\mathbf{x x}}(h)\right|<\infty$, a matriz de densidade espectral $\mathbf{f}_{\mathbf{x x}}(\lambda)$ para o vetor de séries $\mathbf{X}_{t}=\mathbf{X}_{1 t}-i \mathbf{X}_{2 t}$, será dada por:

$$
\mathbf{f}_{\mathbf{x x}}(\lambda)=\sum_{h=-\infty}^{\infty} \boldsymbol{\Gamma}_{\mathbf{x x}}(h) \exp (-2 \pi h \lambda i)
$$

em que $\mathbf{f}_{\mathbf{x x}}(\lambda)$ é uma matriz complexa $(p \times p)$, não negativa definida e Hermitiana, ou seja, matriz quadrada de elementos complexos de tal forma que a sua transposta conjugada é igual a ela mesma.

A análise de componentes principais para o domínio da frequência será similar à análise de componentes principais do caso clássico, uma vez que a matriz de densidade espectral $\mathbf{f}_{\mathbf{x x}}(\lambda)$ substitui, para o domínio da frequência, a funcionalidade da matriz de covariância $\boldsymbol{\Sigma}_{\mathbf{x}}$ do caso clássico.

No caso clássico observamos $p$ variáveis $\mathbf{X}=\left(X_{1}, \ldots, X_{p}\right)^{\prime}$ e encontramos, por meio de combinações lineares, as componentes $\mathbf{Y}=\left(Y_{1}, \ldots, Y_{p}\right)$ não correlacionadas e que descrevem a variação dos dados observados $\mathbf{X}$

$$
\mathbf{Y}=\mathbf{c}^{\prime} \mathbf{X}=c_{1} X_{1}+c_{2} X_{2}+\ldots+c_{p} X_{p}
$$

Como vimos, podemos encontrar as componentes principais, efetuando a maximização

$$
\max _{\mathbf{c} \neq 0} \frac{\mathbf{c}^{\prime} \Sigma_{\mathbf{x}} \mathbf{c}}{\mathbf{c}^{\prime} \mathbf{c}}, \mathbf{c}^{\prime} \mathbf{c}=1
$$


em que $\boldsymbol{\Sigma}_{\mathbf{x}}$ é a matriz de covariância de $\mathbf{X}$. Neste caso, para a primeira componente, teremos $\mathbf{c}=\mathbf{e}_{\mathbf{1}}$, em que $\mathbf{e}_{\mathbf{1}}$ é o autovetor correspondente ao maior autovalor $\lambda_{1}$, então:

$$
\frac{\mathbf{e}_{1}^{\prime} \Sigma_{\mathbf{x}} \mathbf{e}_{\mathbf{1}}}{\mathbf{e}_{\mathbf{1}}^{\prime} \mathbf{e}_{\mathbf{1}}}=\lambda_{1} \text { para } \mathbf{e}_{\mathbf{1}}^{\prime} \mathbf{e}_{\mathbf{1}}=1
$$

Assim, de forma similar ao caso clássico, encontramos as componentes para o domínio da frequência da seguinte forma:

$$
\max _{\mathbf{c}(\lambda) \neq 0} \frac{\mathbf{c}(\lambda)^{*} \mathbf{f}_{\mathbf{x x}}(\lambda) \mathbf{c}(\lambda)}{\mathbf{c}(\lambda)^{*} \mathbf{c}(\lambda)}, \text { para } \mathbf{c}(\lambda)^{*} \mathbf{c}(\lambda)=1
$$

em que $\mathbf{f}_{\mathbf{x x}}(\lambda)$ é a matriz de densidade espectral. Com isso, determinamos para uma frequência fixa $\lambda$ o total de $p$ componentes. Assim, como resultado da maximização acima, determinamos os respectivos pares de autovalores e autovetores de $\mathbf{f}_{\mathbf{x x}}(\lambda)\left(w_{1}(\lambda), \mathbf{e}_{\mathbf{1}}(\lambda)\right), \ldots,\left(w_{p}(\lambda), \mathbf{e}_{\mathbf{p}}(\lambda)\right)$, em que $w_{1}(\lambda) \geq w_{2}(\lambda) \geq \ldots \geq w_{p}(\lambda) \geq 0$.

Desta forma, temos para um determinado valor de frequência $\lambda$, a seguinte relação para o primeiro autovetor e autovalor de $\mathbf{f}_{\mathbf{x x}}(\lambda)$.

$$
\max _{\mathbf{c}(\lambda) \neq 0} \frac{\mathbf{c}(\lambda)^{*} \mathbf{f}_{\mathbf{x x}}(\lambda) \mathbf{c}(\lambda)}{\mathbf{c}(\lambda)^{*} \mathbf{c}(\lambda)}=\frac{\mathbf{e}_{\mathbf{1}}(\lambda)^{*} \mathbf{f}_{\mathbf{x x}}(\lambda) \mathbf{e}_{\mathbf{1}}(\lambda)}{\mathbf{e}_{\mathbf{1}}(\lambda)^{*} \mathbf{e}_{\mathbf{1}}(\lambda)}=w_{1}(\lambda) .
$$

Este processo pode ser repetido para todo valor de frequência $\lambda$ até a $p$-ésima componente principal.

\subsection{Estimador para a Matriz de Densidade Espectral e Escolha do Parâmetro da Frequência $\lambda$}

Como vimos, para um dado valor de frequência, podemos determinar a matriz de densidade espectral da seguinte forma:

$$
\mathbf{f}_{\mathbf{x x}}(\lambda)=\sum_{h=-\infty}^{\infty} \boldsymbol{\Gamma}_{\mathbf{x x}}(h) \exp (-2 \pi h \lambda i)
$$


Contudo, na descrição sobre análise espectral vimos que podemos utilizar o periodograma como estimador para a densidade espectral. Assim, podemos estimar a matriz de densidade espectral $\mathbf{f}_{\mathbf{x x}}(\lambda)$ da seguinte forma:

$$
\hat{\mathbf{f}_{\mathbf{x x}}}(\lambda)=\sum_{l=-m}^{m} h_{l} \mathbf{I}_{\mathbf{N}}(\lambda+l / N),
$$

em que $\mathbf{I}_{\mathbf{N}}(\lambda)=\frac{1}{2 \pi} \sum_{h=-[N-1]}^{[N-1]} \hat{\boldsymbol{\Gamma}_{\mathbf{x x}}}(h) \exp (-i h \lambda)$ e $\hat{\boldsymbol{\Gamma}_{\mathbf{x x}}}(h)=\left[\gamma_{i j}(h)\right]=\frac{1}{N} \sum_{t=1}^{N-|h|} X_{i t} X_{j(t+|h|)}$, quando $E\left(X_{i t}\right)=E\left(X_{j t}\right)=0$ para $i, j=1, \ldots, p$.

O total de parcelas $(2 m+1)$ é ímpar e os pesos são escolhidos de tal forma que $h_{l}=h_{-l}$ sejam positivos e $\sum_{l} h_{l}=1$.

A escolha do valor da frequência é convenientemente escolhido pelos estimadores de espectros determinados pelo periodograma. Assim, quanto maior o estimador para uma dada frequência $\lambda$, maior será a variância dos dados atribuída à frequência.

\subsection{Aplicação da Análise de Componentes Principais no Domínio da Frequência aos Dados fMRI}

Neste exemplo, utilizamos os mesmos dados em que aplicamos a análise de componentes principais pelo método clássico, relembrando que foram medidos o nível de oxigenação do sangue após estímulos aplicados a diferentes localidades do cérebro.

Para o estudo, contamos com 8 variáveis $\mathbf{X}_{\mathbf{t}}=\left(X_{1 t}, X_{2 t}, X_{3 t}, X_{4 t}, X_{5 t}, X_{6 t}, X_{7 t}, X_{8 t}\right)$ que representam medidas consecutivas do nível de oxigenação do sangue segundo as $N$ unidades de tempo.

Para a análise no domínio da frequência precisamos, com base nos dados, encontrar a matriz de densidade espectral $\hat{\mathbf{f}_{\mathbf{x x}}}(\lambda)$ e definirmos o valor de frequência $\lambda$.

Com o objetivo de encontrar a frequência $\lambda$, calculamos o periodograma alisado para cada uma das séries da seguinte forma:

$$
\hat{\mathbf{f}_{\mathbf{x x}}}(\lambda)=\sum_{l=-m}^{m} h_{l} \mathbf{I}_{\mathbf{N}}(\lambda+l / N),
$$


em que os pesos são escolhidos tais que $h_{l}=h_{-l}$ seja positivo e $\sum_{l} h_{l}=1$.

Neste exemplo fixamos os seguintes valores para $h_{l}: h_{0}=3 / 9, h_{1}=2 / 9, h_{2}=1 / 9$.

A Figura 4.1 mostra o periodograma para as 8 variáveis.

Observa-se que, para praticamente todos os periodogramas, exceto para as variáveis $X_{6 t}, X_{7 t}$, identificamos um alto valor de espectro para o ciclo=4, ou seja $\lambda=4 / 128$. Assim, identificamos o parâmetro para estimar $\mathbf{f}_{\mathbf{x} \mathbf{x}}(\lambda)$. Necessitamos agora, para determinar as componentes principais, do valor de $\mathbf{c}(4 / 128)$, tal que:

$$
\max _{\mathbf{c}(4 / 128) \neq 0} \frac{\mathbf{c}(4 / 128)^{*} \mathbf{f}_{\mathbf{x x}}(4 / 128) \mathbf{c}(4 / 128)}{\mathbf{c}(4 / 128)^{*} \mathbf{c}(4 / 128)}, \mathbf{c}(4 / 128)^{*} \mathbf{c}(4 / 128)=1
$$

Para $\lambda=4 / 128$, encontramos para a primeira componente a explicação de $96 \%$ da variabilidade.

As cargas do autovetor para a primeira componente $\hat{e}_{1}\left(\frac{4}{128}\right)$, segundo as localizações $(1, . ., 8)$, são respectivamente: $(0,47 ; 0,37 ; 0,34 ; 0,28 ; 0,40 ;-0,06 ; 0,17 ; 0,44)$.

Na Figura 4.2, mostramos o maior autovalor $w_{1}(\lambda)$ associado a cada valor de frequência $\lambda, \lambda=i / 128, i=1, \ldots, 64$. Observamos um pico na frequência $12 / 128$ associado predominantemente à variável $X_{7 t}=$ cere 1 que apresenta, pelo teste de periodicidade de Fisher [1929] , periodicidade para frequência $\lambda=12 / 128$ com nível de significancia de $95 \%$. Mais detalhes sobre testes para periodicidades pode ser encontrado em Morettin e Toloi [2006].

As cargas do autovetor para a primeira componente $\hat{e}_{1}\left(\frac{12}{128}\right)$ são respectivamente $(0,21 ; 0,18$; $0,34 ; 0,08 ; 0,01 ; 0,44 ; 0,61 ; 0,24)$.

Os valores estimados da primeira componente para as frequências $\lambda=4 / 128$ e $\lambda=12 / 128$, são respectivamente:

$$
\begin{gathered}
Y_{t 1}(4 / 128)=\mathbf{e}_{\mathbf{1}}{ }^{*}(4 / 128) \mathbf{X}_{\mathbf{t}} \\
Y_{t 1}(12 / 128)=\mathbf{e}_{\mathbf{1}}{ }^{*}(12 / 128) \mathbf{X}_{\mathbf{t}}
\end{gathered}
$$

As primeiras componentes principais para $\lambda=4 / 128$ e $\lambda=12 / 128$ estão representadas respectivamente nas Figuras 4.3 e 4.4 . 

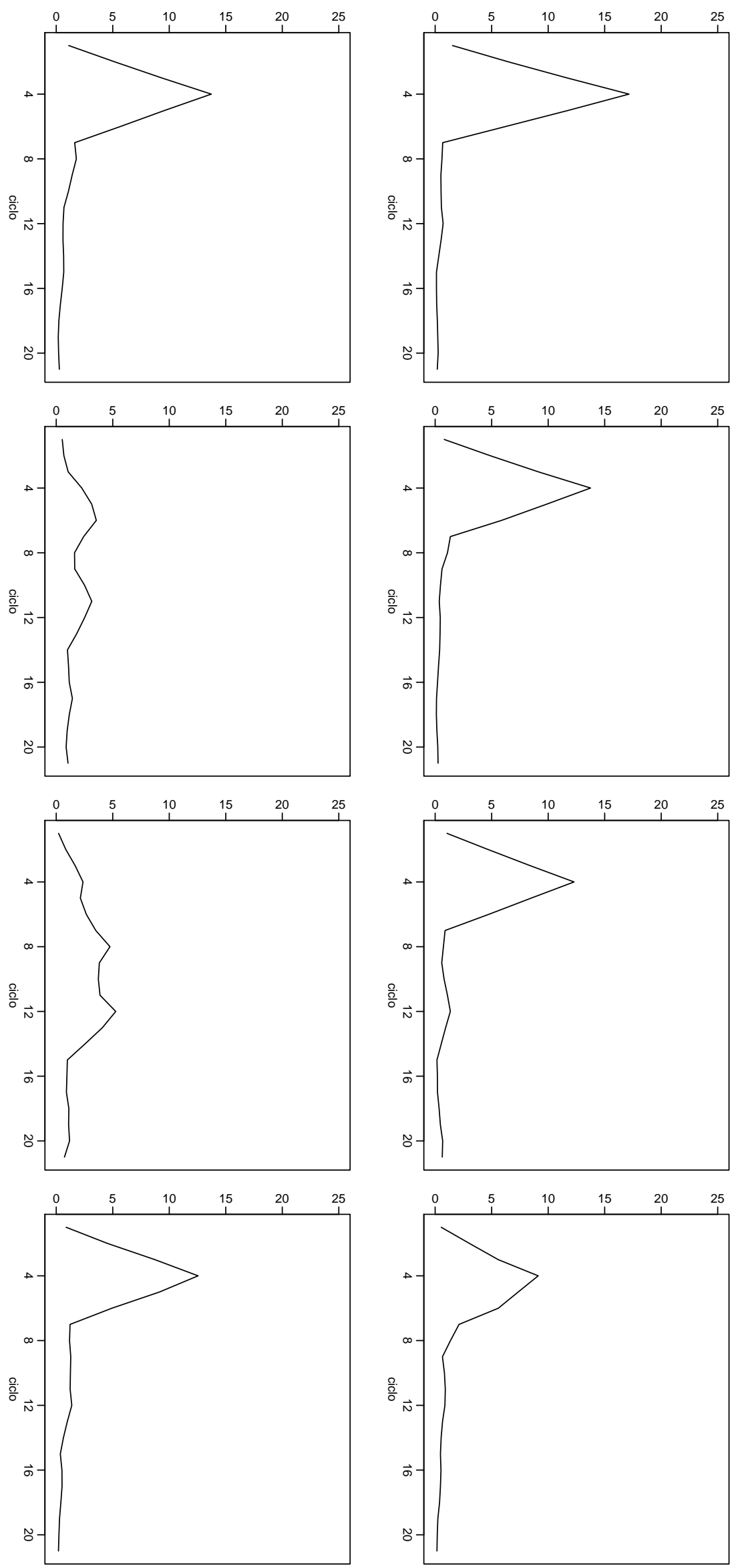

Figura 4.1: Periodograma alisado para os dados observados fMRI: $X_{1 t}=$ cort1,$X_{2 t}=$ cort2, $X_{3 t}=$ cort3, $X_{4 t}=$ cort $4, X_{5 t}=$ thal1,$X_{6 t}=$ thal2, $X_{7 t}=$ cere $1, X_{8 t}=$ cere2 
APLICAÇÃO DA ANÁLISE DE COMPONENTES PRINCIPAIS NO DOMÍNIO DA FREQUÊNCIA 4.3

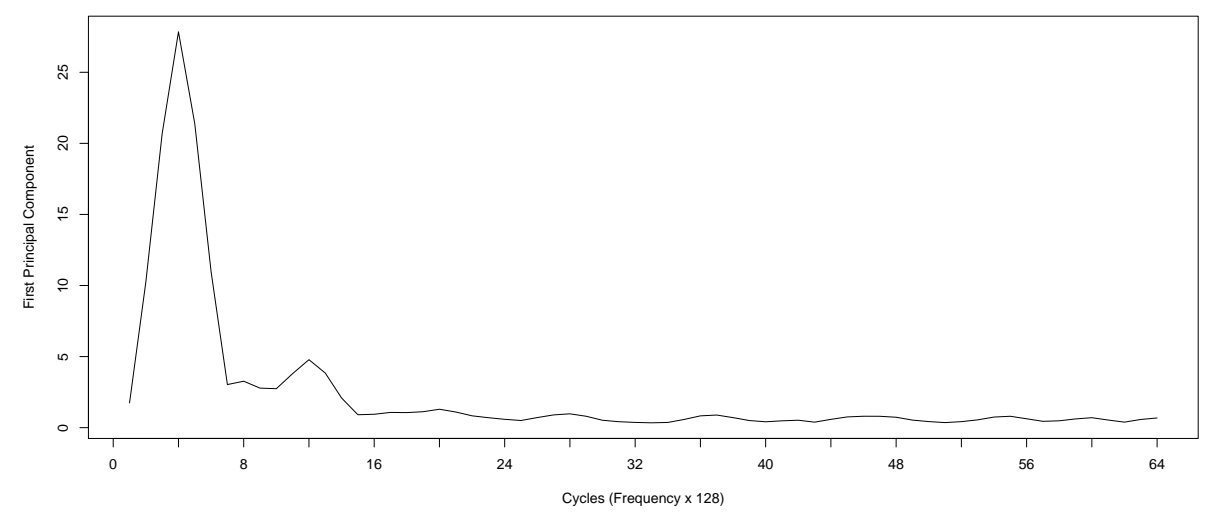

Figura 4.2: Maior autovalor associado a cada ciclo ou frequência $\lambda=i / 128$

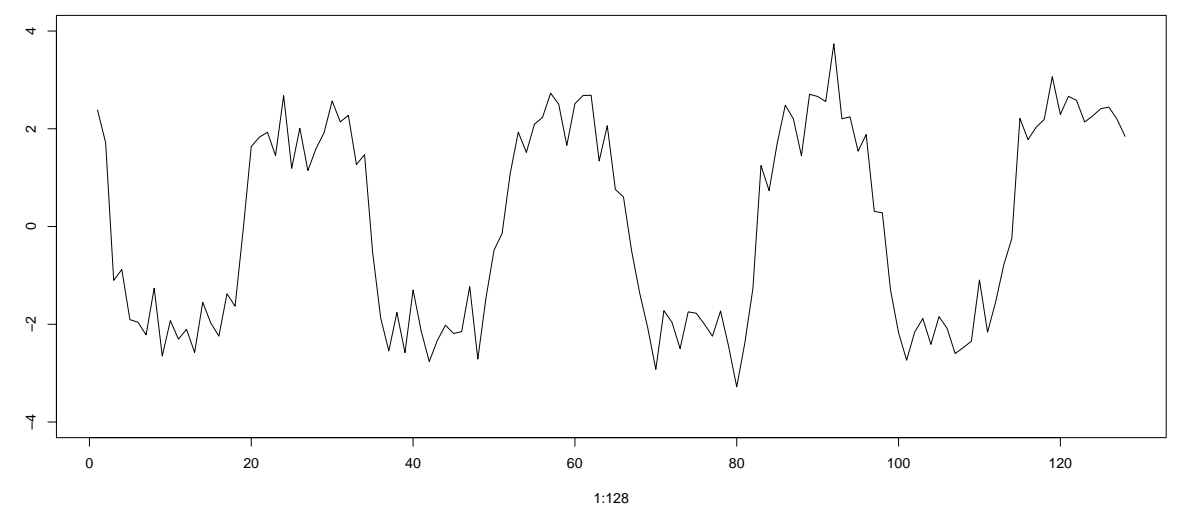

Figura 4.3: Primeira componente para a frequência (4/128)

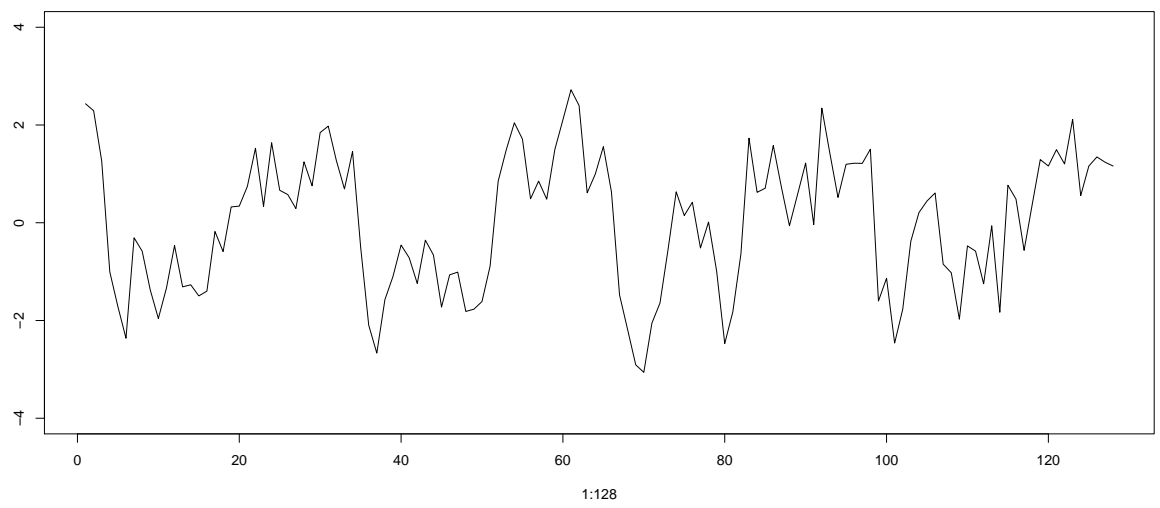

Figura 4.4: Primeira componente para a frequência (12/128) 


\subsection{Sobre os Dados e Resultados}

$\mathrm{Na}$ análise de componentes principais do caso clássico, encontramos na primeira componente uma forte estrutura periódica dada pelo efeito de resposta ao estímulo a cada intervalo regular de tempo. Desta forma, as cargas da primeira componente foram $(0,45 ; 0,40 ; 0,41$; $0,32 ; 0,38 ;-0,07 ; 0,21 ; 0,38)$.

Quando analisamos o mesmo conjunto de dados pelo domínio da frequência conseguimos, por meio do periodograma, identificar de forma mais específica a estrutura periódica dada pela intensidade de resposta de cada uma das variáveis ao estímulo.

Quando escolhemos o maior espectro, ou seja frequência $\lambda=4 / 128$, estamos analisando exatamente a frequência com que foi aplicado o estímulo, ou seja 1 a cada 32 segundos ou 4 em 128.

Com isso, o resultado esperado para o domínio da frequência, com $\lambda=4 / 128$, deve ser similar ao resultado da primeira componente do caso clássico com baixa carga para as variáveis $X_{6 t}$ e $X_{7 t}$, como de fato obtivemos.

Por outro lado, analisando a frequência $\lambda=12 / 128$, encontramos altas cargas para as variáveis $X_{6 t}$ e $X_{7 t}$, indicando que predominantemente a resposta ao estímulo, para estas duas localidades do cérebro, ocorre com frequência e intensidade diferentes das demais regiões do cérebro.

\subsection{Método Clássico $\times$ Domínio da Frequência}

A principal vantagem em utilizarmos a análise de componentes principais no domínio da frequência consiste na possibilidade de gerar diferentes componentes para diferentes frequências, fornecendo um resultado mais elaborado e específico.

No caso clássico, obtivemos o efeito e comportamento de uma forma mais geral. Por outro lado, séries que possuem praticamente um valor de espectro muito significativo para uma única frequência $\lambda$, como vimos no exemplo acima, não devem apresentar mudanças representativas entre a análise pelo domínio da frequência ou pelo método clássico.

No exemplo acima, tanto para domínio da frequência quanto para o método clássico, os resultados encontrados foram similares, contudo, Lagerlund et al. [2004] mostra que uma li- 
mitação para o algoritmo clássico de componentes principais é que duas ou mais variáveis com diferentes frequências, com similar amplitude podem ser extraídas através de uma mesma componente, tornando-se impossível separá-las. Desta forma, Lagerlund et al. [2004] argumenta que uma análise apropriada de componentes principais no domínio da frequência potencializa o resultado. No exemplo apresentado acima todas as séries apresentam praticamente o domínio pela frequência 4/128, por este motivo os resultados encontrados são similares. 


\section{Capítulo 5}

\section{SSA Singular Spectrum Analysis}

O método SSA é uma análise derivada da análise de componentes principais, com o objetivo de decompor uma série de tempo $X_{t}$ em componentes aditivas que representam padrões da série de tempo como: tendência, oscilação, periodicidade e ruído. A análise envolve o uso de autovalores e autovetores de uma matriz de covariância construída a partir da série de tempo $X_{t}$ e um parâmetro chamado tamanho da janela, que indicamos por $M$.

Veremos mais adiante que o tamanho da janela $M$ depende de informações preliminares sobre a série de tempo e do tipo de decomposição desejada como: tendência, sazonalidade, etc.

Descrevemos o método de análise SSA em duas etapas complementares, a primeira chamada de decomposição e a segunda de reconstrução. Mais detalhes sobre o método pode ser entrado em Wilks [2011], Jolliffe [2002], Golyandina et al. [2001].

De forma simplificada, na etapa de decomposição, iniciamos construíndo a matriz de trajetórias $\mathbf{H}$ que consiste em representar uma série de tempo unidimensional $X_{t}=\left(X_{1}, \ldots, X_{N}\right)^{\prime}$ em uma série multidimensional $\mathbf{H}_{(1)}, \mathbf{H}_{(2)}, \ldots, \mathbf{H}_{(K)}$ para $K=N-M+1$. Em seguida, fazemos a decomposição do valor singular no qual escrevemos a matriz de trajetórias $\mathbf{H}$ em componentes aditivas.

Na etapa de reconstrução trabalhamos com as componentes aditivas com o objetivo de reagrupá-las e determinarmos padrões e estruturas da série observada $X_{t}$. Supondo, como exemplo, que a série de tempo observada $X_{t}$ tenha em sua estrutura um efeito de periodicidade dado por $W_{1 t}$ e um ruído aleatório dado $W_{2 t}$, podemos escrever $X_{t}=W_{1 t}+W_{2 t}$. 
Desejamos reconstruir a série de tempo com a identificação de dois grupos um associado à periodicidade $W_{1 t}$ e um outro ao ruído $W_{2 t}$.

A seguir, descrevemos com mais detalhes cada uma das duas etapas e mostramos um exemplo da aplicação para uma série de fMRI.

\subsection{Primeira Etapa: Decomposição}

Começamos com a contrução da matriz de trajetórias que consiste em representar uma série de tempo $X_{t}, t=1, \ldots, N$ em séries multimensionais de dimensão $M \times(N-M+1)$, em que $M$ é o tamanho da janela e tem para a análise uma grande importância, pois define a dimensão da matriz de covariância e a quantidade de componentes principais determinadas. Dada uma série de tempo $X_{t}, t=1, \ldots, N$,(padronizada), definimos $\mathbf{H}$ como a matriz de trajetórias do vetor da série de tempo $X_{t}=\left(X_{1}, X_{2}, \ldots, X_{N}\right)^{\prime}$, em que o elemento $h_{t}$ representado abaixo, é o elemento de $X$ no instante de tempo $t$, ou seja $X_{t}$.

$$
\begin{gathered}
\mathbf{H}=\mathbf{H}_{(1)}^{\prime}, \mathbf{H}_{(2)}^{\prime}, \ldots, \mathbf{H}_{(N-M+1)}^{\prime}, \text { em que } \\
\mathbf{H}_{(1)}=\left(h_{1}, h_{2}, \ldots, h_{M}\right) \\
\mathbf{H}_{(2)}=\left(h_{2}, h_{3}, \ldots, h_{M+1}\right) \\
\vdots \\
\mathbf{H}_{(N-M+1)}=\left(h_{N-M+1}, h_{N-M+2}, \ldots, h_{N}\right)
\end{gathered}
$$

$\mathrm{Ou}$

$$
\mathbf{H}=\left[\begin{array}{ccccc}
h_{1} & h_{2} & h_{3} & \cdots & h_{N-M+1} \\
h_{2} & h_{3} & h_{4} & \cdots & h_{N-M+2} \\
h_{3} & h_{4} & h_{5} & \cdots & h_{N-M+3} \\
\vdots & \vdots & \vdots & \ddots & \vdots \\
h_{M} & h_{M+1} & h_{M+2} & \cdots & h_{N}
\end{array}\right]
$$


em que $M$ é inteiro definido como tamanho da janela, $1<M<N$ e $K=N-M+1$. Assim, a matriz de trajetórias $\mathbf{H}$ tem dimensão $(M \times K)$ e $\mathbf{H}$ representa uma matriz do tipo Hankel, com elementos $h_{i, j}=h_{i-1, j+1}, i=2, \ldots, M$ e $j=1, \ldots, N-1$.

Uma vez calculada a matriz $\mathbf{H}$, construímos a matriz $\mathbf{S}=\left(s_{i j}\right)=\operatorname{cov}\left(\mathbf{H}_{(i)}, \mathbf{H}_{(j)}\right)$. Supondo $X_{t}$ estacionária, a matriz $\mathbf{S}$ representa uma matriz do tipo Toeplitz com $\left[s_{i j}\right]=\left[\gamma_{|i-j|}\right]=E\left[X_{t} X_{t+|i-j|}\right]$. Assim, para $M$ fixo, os elementos da matriz dependem somente da distância $|i-j|$ para $i, j=1, \ldots, M$.

$$
\mathbf{S}=\left[\begin{array}{cccc}
s_{11} & s_{12} & \cdots & s_{1 M} \\
s_{21} & s_{22} & \cdots & s_{2 M} \\
\vdots & \vdots & \ddots & \vdots \\
s_{M 1} & s_{M 2} & \cdots & s_{M M}
\end{array}\right]
$$

Denotamos por $\lambda_{1}, \lambda_{2}, \ldots, \lambda_{M}$ os autovalores e $\mathbf{U}_{1}, \mathbf{U}_{2}, \ldots, \mathbf{U}_{M}$ os autovetores, respectivamente, da matriz $\mathbf{S}$ e $d=\max \left[i\right.$, tal que $\left.\lambda_{i}>0\right]$. Com isso, se denotarmos $\mathbf{V}_{i}=\mathbf{H}^{\prime} \mathbf{U}_{i} / \sqrt{\lambda}_{i}$ $(i=1, \ldots, d)$, podemos escrever a matriz de trajetórias $\mathbf{H}$ em componentes aditivas:

$$
\mathbf{H}=\mathbf{H}_{I_{1}}+\ldots+\mathbf{H}_{I_{d}}
$$

em que $\mathbf{H}_{I_{i}}=\sqrt{\lambda_{i}} \mathbf{U}_{i} \mathbf{V}_{i}^{\prime}$. Desta forma, temos a decomposição do valor singular (Golyandina et al. [2001]), da matriz $\mathbf{H}$ com as combinações dos valores $\left(\sqrt{\lambda}_{i}, \mathbf{U}_{i}, \mathbf{V}_{i}\right)$ denominado autotriplo da decomposição.

\subsection{Segunda Etapa: Reconstrução}

Nesta etapa, desejamos reconstruir $r$ novas séries, em que cada uma estará associada a uma característica ou estrutura da série de tempo $X_{t}$.

Construímos os respectivos grupos, agrupando devidamente os resultados da decomposição do valor singular da matriz $\mathbf{H}$ :

$$
\mathbf{H}=\mathbf{H}_{I_{1}}+\ldots+\mathbf{H}_{I_{d}} .
$$


O principal desafio consiste em agrupar os valores de $\mathbf{H}_{I_{i}}, i=1, \ldots, d$, em $r$ partes, $\mathbf{H}^{(1)}$, $\mathbf{H}^{(2)}, \ldots, \mathbf{H}^{(r)}$, em que $\mathbf{H}^{(i)}$ tem dimensão $(M \times(N-M+1))$ e está associada a uma característica da série de tempo $X_{t}$. Teremos um excelente resultado se cada uma das partes $\mathbf{H}^{(1)}, \mathbf{H}^{(2)}, \ldots, \mathbf{H}^{(r)}$ tiver uma estrutura próxima de uma matriz de Hankel.

Construímos $\mathbf{H}^{(i)}$, da seguinte forma:

$$
\begin{gathered}
\mathbf{H}^{(1)}=\sum_{i=1}^{d_{1}} \mathbf{H}_{I_{i}} \\
\mathbf{H}^{(2)}=\sum_{i=d_{1}+1}^{d_{2}} \mathbf{H}_{I_{i}} \\
\vdots \\
\mathbf{H}^{(r)}=\sum_{i=d_{r-1}+1}^{d} \mathbf{H}_{I_{i}} .
\end{gathered}
$$

Temos como consequência $r$ grupos, $r \leq d$, tais que $\mathbf{H}=\mathbf{H}^{(1)}+\mathbf{H}^{(2)}+\ldots+\mathbf{H}^{(r)}$.

Assim, podemos dizer que a série $X_{t}$ pode ser explicada por $r$ características dadas por $\left(\mathbf{H}^{(1)}, \ldots, \mathbf{H}^{(r)}\right)$, podemos usar como medida de explicação de cada um dos $\mathbf{H}^{(i)}$ a soma dos autovalores das $\mathbf{H}_{I_{i}}$ utilizados para a construção de $\mathbf{H}^{(i)}$ sobre a soma de todos $d$ autovalores, ou seja, teríamos para $\mathbf{H}^{(1)}: \sum_{i=1}^{d_{1}} \lambda_{i} / \sum_{i=1}^{d} \lambda_{i}$.

Após construírmos $\mathbf{H}^{(1)}, \mathbf{H}^{(2)}, \ldots, \mathbf{H}^{(r)}$, vamos transformar cada uma das $\mathbf{H}^{(i)}$ em um vetor com $N$ elementos utilizando a diagonalização de $\mathbf{H}^{(i)}$, que nada mais é que a etapa inversa da construção da matriz de trajetórias $\mathbf{H}$.

$$
\mathbf{H}^{(i)}=\left[\begin{array}{ccccc}
h_{1,1}^{(i)} & h_{1,2}^{(i)} & h_{1,3}^{(i)} & \cdots & h_{1, K}^{(i)} \\
h_{2,1}^{(i)} & h_{2,2}^{(i)} & h_{2,3}^{(i)} & \cdots & h_{2, K}^{(i)} \\
h_{3,1}^{(i)} & h_{3,2}^{(i)} & h_{3,3}^{(i)} & \cdots & h_{3, K}^{(i)} \\
\vdots & \vdots & \vdots & \ddots & \vdots \\
h_{M, 1}^{(i)} & h_{M, 2}^{(i)} & h_{M, 3}^{(i)} & \cdots & h_{M, K}^{(i)}
\end{array}\right]
$$


em que $K=N-M+1$.

Portanto, teremos respectivamente para cada $\mathbf{H}^{(i)}$, o seguinte vetor $\widetilde{\mathbf{H}^{(i)}}$ :

$$
\widetilde{\mathbf{H}^{(i)}}=\left[\begin{array}{c}
h_{1,1}^{(i)} \\
\frac{h_{1,2}^{(i)}+h_{2,1}^{(i)}}{2} \\
\frac{h_{1,3}^{(i)}+h_{2,2}^{(i)}+h_{3,1}^{(i)}}{3} \\
\vdots \\
\frac{h_{M-2, K}^{(i)}+h_{M-1, K-1}^{(i)}+h_{M, K-2}^{(i)}}{3} \\
\frac{h_{M-1, K}^{(i)}+h_{M, K-1}^{(i)}}{2} \\
h_{M, K}^{(i)}
\end{array}\right]
$$

em que $\widetilde{\mathbf{H}^{(i)}}$ tem dimensão $(N \times 1)$, e para cada $i$ pode representar uma estrutura como tendência, periodicidade ou ruído da série de tempo $X_{t}$.

Como exemplo, consideramos $\mathrm{N}=6, \mathrm{M}=4$ e $\mathrm{K}=3$

$$
\mathbf{H}^{(i)}=\left[\begin{array}{ccc}
h_{1,1}^{(i)} & h_{1,2}^{(i)} & h_{1,3}^{(i)} \\
h_{2,1}^{(i)} & h_{2,2}^{(i)} & h_{2,3}^{(i)} \\
h_{3,1}^{(i)} & h_{3,2}^{(i)} & h_{3,3}^{(i)} \\
h_{4,1}^{(i)} & h_{4,2}^{(i)} & h_{4,3}^{(i)}
\end{array}\right]
$$


e

$$
\widetilde{\mathbf{H}^{(i)}}=\left[\begin{array}{c}
h_{1,1}^{(i)} \\
\frac{h_{1,2}^{(i)}+h_{2,1}^{(i)}}{2} \\
\frac{h_{1,3}^{(i)}+h_{2,2}^{(i)}+h_{3,1}^{(i)}}{3} \\
\frac{h_{2,3}^{(i)}+h_{3,2}^{(i)}+h_{4,1}^{(i)}}{3} \\
\frac{h_{4,2}^{(i)}+h_{3,3}^{(i)}}{2} \\
h_{4,3}^{(i)}
\end{array}\right] .
$$

Em muitos casos, estamos procurando um padrão específico para $X_{t}$. Neste caso, avaliamos os maiores autovalores calculados e determinamos se a matriz dos respectivos $\mathbf{H}_{I_{i}}$, com maiores autovalores, seguem uma estrutura próxima de uma matriz de Hankel. Outra análise muito importante, consiste em utilizar o periodograma da matriz $\widetilde{\mathbf{H}^{(i)}}$. No exemplo deste Capítulo utilizamos, para dados fMRI, o periodograma na análise dos grupos formados.

\subsection{SSA e Análise de Fourier}

Assim como na análise de Fourier, o método SSA detecta e representa efeitos oscilatórios da série de tempo. A periodicidade, por exemplo, pode ser representada na SSA pelas correspondentes cargas dos autovetores. A análise das cargas pode revelar comportamentos similares a funções trigonométricas (senos e cossenos), contudo uma das motivações para usar SSA é que o comportamento observado pelos autovetores refletem padrões da série de tempo. Em SSA podemos estar interessados em efeitos não senoidais e além disso SSA pode representar variações temporais, mudanças estruturais da série ao longo do tempo e que não são como na análise de Fourier recorrentes ao longo do tempo. 


\subsection{Escolha do Tamanho da Janela $M$}

Uma importante decisão na análise de SSA é a escolha do tamanho da janela $M$. Não existe uma regra para definir previamente e objetivamente o tamanho da janela, entretanto informações preliminares da série, bem como a estrutura que se deseja extrair pode colaborar para a escolha do tamanho da janela.

Em geral o tamanho da janela $M$ é menor que $N / 3$, contudo Golyandina et al. [2001] argumenta que obtemos informações mais detalhadas da série $X_{t}$ quando $M$ se aproxima de $N / 2$, mas a análise pode se tornar inviável quando $M$ se torna muito grande, dado o volume de informações. Com isso, poderíamos ter problemas e possivelmente encontrar que nenhuma das $K$-ésimas componentes ajudem a obter uma boa decomposição da série.

Por outro lado, se $M$ for muito pequeno, podemos encontrar uma baixa qualidade na decomposição da série, ou seja, $M$ pequeno pode não separar adequadamente os efeitos e estruturas da série de tempo.

Para uma série de tempo com estrutura não muito complexa, um grande tamanho de janela pode produzir indesejadas decomposições. Assim poderíamos gerar grupos $\mathbf{H}^{(i)}$ inadequados, ou seja, não refletindo adequadamente a estrutura desejada.

A seguir exploramos desafios na escolha do tamanho da janela para algumas estruturas particulares da série de tempo.

\subsubsection{Escolha do Tamanho da Janela para Extração de Tendência}

Para extração de tendência, precisamos definir um alto valor do tamanho da janela $M$, mas existem contradições em identificar fraca e forte decomposição da série $X_{t}$.

Golyandina et al. [2001] fornece um exemplo de aniversários em que a janela é de ordem 1000, uma vez que as componentes de tendências estão misturadas com componentes periódicas semestrais.

Para uma mistura muito complexa de $\mathbf{H}=\mathbf{H}^{(1)}+\mathbf{H}^{(2)}$, com $\mathbf{H}^{(1)}$ tendência e $\mathbf{H}^{(2)}$ ruído aleatório, teremos muitos problemas para separá-los exigindo um tamanho de janela bem elevado. Contudo, para séries menos complexas a separação será mais simples e exigirá uma quantidade inferior no tamanho da janela. 


\subsubsection{Escolha do Tamanho da Janela para Alisamento de Curva}

No caso de suavização da curva, podemos pensar em associar a análise com análise espectral da série, de tal forma que seja possível fazer a separação entre os espectros de baixas e altas frequências da série $X_{t}$.

Podemos com isso estabelecer um valor $M$ e analisar o periodograma do resíduo, verificando

se todo comportamento espectral da série $X_{t}$ foi explicado, por exemplo, por $\widetilde{\mathbf{H}^{(i)}}$. Um problema pode existir quando não for possível separar o maior do menor espectro da série na decomposição.

\subsubsection{Escolha do Tamanho da Janela para Séries Periódicas}

O desafio de escolher o tamanho da janela $M$ para séries periódicas envolve certas particularidades relacionadas ao tamanho da janela $M$ e o período da série $T$.

Assim como na identificação do tamanho da janela para alisamento de curva, identificaremos o tamanho da janela $M$ avaliando o periodograma de $X_{t}$.

De forma geral para séries não muito longas, a escolha de $M$ é tal que a razão entre $M / T$ seja inteira. Para séries longas, se existir facilidade de análise é recomendado trabalhar com $M$ próximo de $N / 2$.

Golyandina et al. [2001] recomenda que para séries longas $M / T$ seja inteiro, mesmo que não seja necessário.

\subsubsection{Escolha do Tamanho da Janela para Séries Desconhecidas}

Quando não temos informações sobre a série original, ou seja, nenhuma informação pode ser usada para identificar o tamanho da janela, a recomendação é trabalhar com a análise do periodograma da série original $X_{t}$, para conhecermos melhor a estrutura e características da série. Assim, através da análise identificaremos importantes informações sobre a estrutura da série, ou seja, identificando o comportamento periódico de $X_{t}$ pelo maiores valores de espectro estimados pelo periodograma. 


\subsection{Quantidade de Grupos para Reconstrução das Séries}

Outra importante decisão consiste na escolha do valor $r$ que está associado à quantidade de grupos formados na etapa de reconstrução. Assim como o tamanho da janela, não existe uma regra para defini-lo objetivamente. Por outro lado, teremos um excelente resultado se cada uma das matrizes $\mathbf{H}^{(1)}, \mathbf{H}^{(2)}, \ldots, \mathbf{H}^{(r)}$ tiver uma estrutura próxima de uma matriz de Hankel.

Segundo Golyandina et al. [2001], uma boa formação de grupos implica que a correlação entre as linhas e colunas das matrizes $\mathbf{H}^{(i)}$ e $\mathbf{H}^{(j)}$ seja próxima de zero para $i, j=1, \ldots, r$ e $i \neq j$.

Para dados periódicos podemos utilizar o periodograma para auxiliar na construção dos grupos. Podemos verificar pelo periodograma se toda periodicidade associada a uma frequência $\lambda$ está contida em apenas um dos $r$ grupos, caso contrário, retornamos para a etapa de formação dos grupos até que a periodicidade observada esteja em um único grupo.

\subsection{Aplicação de SSA para Dados fMRI}

No exemplo usamos os mesmos dados utilizados nos exemplos de componentes principais clássico e análise da análise de componetes principais no domínio da frequência.

No método SSA, toda a análise é feita sobre uma única série de tempo $X_{t}$. Desta forma, neste exemplo, vamos utilizar a série de tempo $X_{t}$, como a medida do nível de oxigenação do sangue na região do cortex dada por $X_{t 1}, t=1 \ldots 128$, O gráfico da Figura 5.1 mostra os dados observados e o respectivo periodograma.

Notamos, na Figura 5.1, uma forte estrutura periódica com período $T$ igual a 128/4=32. Não observamos uma estrutura de tendência, desta forma assumimos $M=32$, conforme sugerimos no item de escolha do tamanho da janela para dados periódicos, ou seja $M / T=1$. Assumindo $M=32$ seguimos a análise construindo a matriz de trajetórias $\mathbf{H}$ que terá di- 

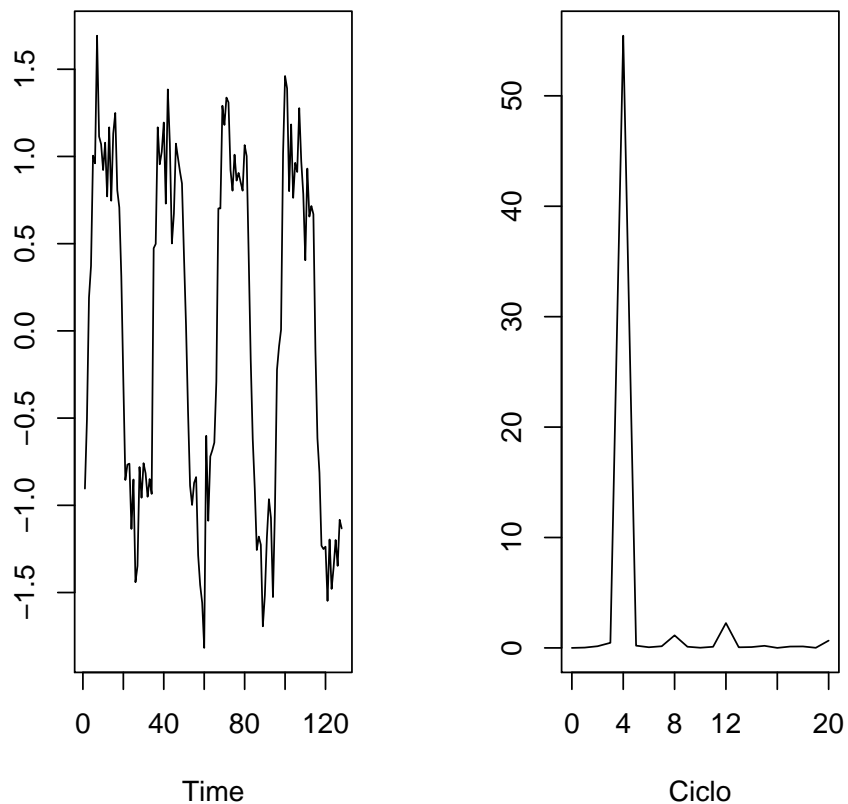

Figura 5.1: A figura da esquerda mostra a série $X_{t}$ e a figura da direita o periodograma

mensão $(M \times(N-M+1))$ ou $(32 \times 97)$, conforme abaixo:

$$
\mathbf{H}=\left[\begin{array}{ccccc}
h_{1} & h_{2} & h_{3} & \cdots & h_{97} \\
h_{2} & h_{3} & h_{4} & \cdots & h_{98} \\
h_{3} & h_{4} & h_{5} & \cdots & h_{99} \\
\vdots & \vdots & \vdots & \ddots & \vdots \\
h_{32} & h_{33} & h_{34} & \cdots & h_{128}
\end{array}\right]
$$

Desta forma a matriz de covariância $\mathbf{S}$ será a estimativa de uma matriz Toeplitz com dimensão $(32 \times 32)$. 


$$
\mathbf{S}=\left[\begin{array}{cccccccc}
0,965 & s_{1,2} & \cdots & \cdots & \cdots & \cdots & \cdots & s_{1,32} \\
0,878 & 0,956 & s_{2,3} & \cdots & \cdots & \cdots & \cdots & s_{2,32} \\
0,792 & 0,873 & 0,963 & s_{3,4} & \cdots & \cdots & \cdots & s_{3,32} \\
0,674 & 0,794 & 0,889 s & 0,984 & s_{4,5} & \cdots & \cdots & s_{4,32} \\
0,537 & 0,677 & 0,809 & 0,908 & 1,01 & s_{5,6} & \cdots & s_{5,32} \\
\vdots & \vdots & \vdots & \vdots & \vdots & \vdots & \ddots & \vdots \\
s_{32,1} & s_{32,2} & s_{32,3} & s_{32,4} & s_{32,5} & s_{32,6} & \cdots & s_{32,32}
\end{array}\right]
$$

A decomposição do valor singular gerou 32 componentes de forma que a matriz de trajetórias $\mathbf{H}$ foi decomposta da seguinte forma:

$$
\mathbf{H}=\mathbf{H}_{I_{1}}+\ldots+\mathbf{H}_{I_{d}}, d=[1, \ldots, 32]
$$

e $\mathbf{H}_{I_{i}}$ tem dimensão $(M \times(N-M+1))$ ou $(32 \times 97)$.

Temos na Figura 5.2, as cargas dos autovetores para os dos 2 resultados mais representativos, $\operatorname{com} \frac{\sum_{i=1}^{2} \lambda_{i}}{\sum_{j=1}^{32} \lambda_{j}}=0,87$

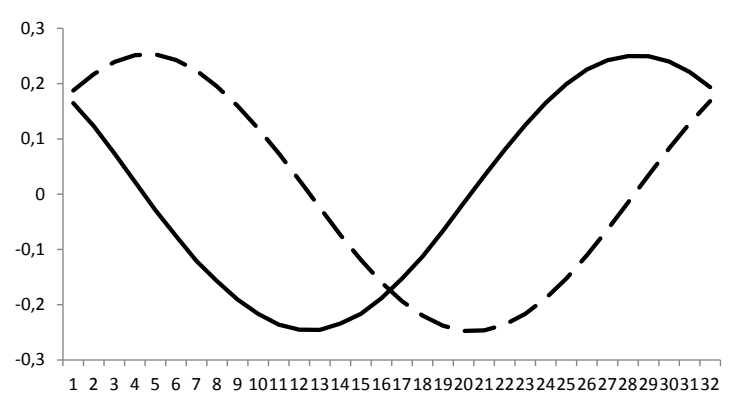

Figura 5.2: cargas dos autovetores para os dois maiores autovalores

Nota-se que $\mathbf{H}_{I_{1}}$ (linha contínua) e $\mathbf{H}_{I_{2}}$ (linha tracejada) têm comportamento senoidal e capturam exatamente o efeito periódico da série $X_{t}$ com T=32 ou 4 ciclos em 128 observações. Os demais $\mathbf{H}_{I_{i}}, i=3 \ldots .32$, representam cada uma das outras características da série, contudo pelo alto espectro da série orginal $X_{t}$ encontrado no ciclo igual 4 direcionamos o foco da análise para $\mathbf{H}_{I_{1}}$ e $\mathbf{H}_{I_{2}}$. 
Notamos que $\mathbf{H}^{(1)}=\mathbf{H}_{I_{1}}+\mathbf{H}_{I_{2}}$ e $\mathbf{H}^{(2)}=\mathbf{H}_{I_{3}}+\ldots+\mathbf{H}_{I_{3} 2}$, seguem a estimativa de uma matriz de Hankel. Os valores das primeiras linhas e colunas de $\mathbf{H}^{(1)}$ e $\mathbf{H}^{(2)}$, são exibidos abaixo.

$$
\mathbf{H}^{(1)}=\left[\begin{array}{ccccccc}
-0,54 & -0,31 & -0,09 & 0,18 & 0,43 & \cdots & h_{1,97}^{(1)} \\
-0,30 & -0,05 & 0,17 & 0,43 & 0,66 & \cdots & h_{2,97}^{(1)} \\
-0,03 & 0,22 & 0,43 & 0,67 & 0,88 & \cdots & h_{3,97}^{(1)} \\
0,24 & 0,48 & 0,68 & 0,89 & 1,06 & \cdots & h_{4,97}^{(1)} \\
0,49 & 0,71 & 0,89 & 1,06 & 1,19 & \cdots & h_{5,97}^{(1)} \\
\vdots & \vdots & \vdots & \vdots & \vdots & \ddots & \vdots \\
h_{32,1}^{(1)} & h_{32,2}^{(1)} & h_{32,3}^{(1)} & h_{32,4}^{(1)} & h_{32,5}^{(1)} & \cdots & h_{32,97}^{(1)}
\end{array}\right]
$$

$$
\mathbf{H}^{(2)}=\left[\begin{array}{ccccccc}
-0,36 & -0,20 & 0,28 & 0,20 & 0,58 & \cdots & h_{1,97}^{(2)} \\
-0,21 & 0,24 & 0,20 & 0,58 & 0,30 & \cdots & h_{2,97}^{(2)} \\
0,22 & 0,15 & 0,57 & 0,29 & 0,82 & \cdots & h_{3,97}^{(2)} \\
0,13 & 0,53 & 0,28 & 0,80 & 0,06 & \cdots & h_{4,97}^{(2)} \\
0,51 & 0,25 & 0,80 & 0,05 & -0,12 & \cdots & h_{5,97}^{(2)} \\
\vdots & \vdots & \vdots & \vdots & \vdots & \ddots & \vdots \\
h_{32,1}^{(2)} & h_{32,2}^{(2)} & h_{32,3}^{(2)} & h_{32,4}^{(2)} & h_{32,5}^{(2)} & \cdots & h_{32,97}^{(2)}
\end{array}\right]
$$

Os gráficos da Figura 5.3 representam as duas estruturas separadas de $X_{t}$, após a diagonalização das matrizes $\mathbf{H}^{(1)}$ e $\mathbf{H}^{(2)}$, com os seus respectivos periodogramas.

Temos que $\mathbf{H}^{(1)}$ é responsável pela principal estrutura da série de tempo $X_{t}$ capturando o comportamento de 4 ciclos em 128 observações e $\mathbf{H}^{(2)}$ capturando os demais 8 e 12 ciclos da série de tempo $X_{t}$. Como mostramos, $\mathbf{H}^{(1)}$ e $\mathbf{H}^{(2)}$ têm estrutura de Hankel e temos com isso uma boa decomposição da série de tempo $X_{t}$ em $\mathbf{H}^{(1)}$, representando o efeito periódico da série e em $\mathbf{H}^{(2)}$, representando os demais efeitos de $X_{t}$. 

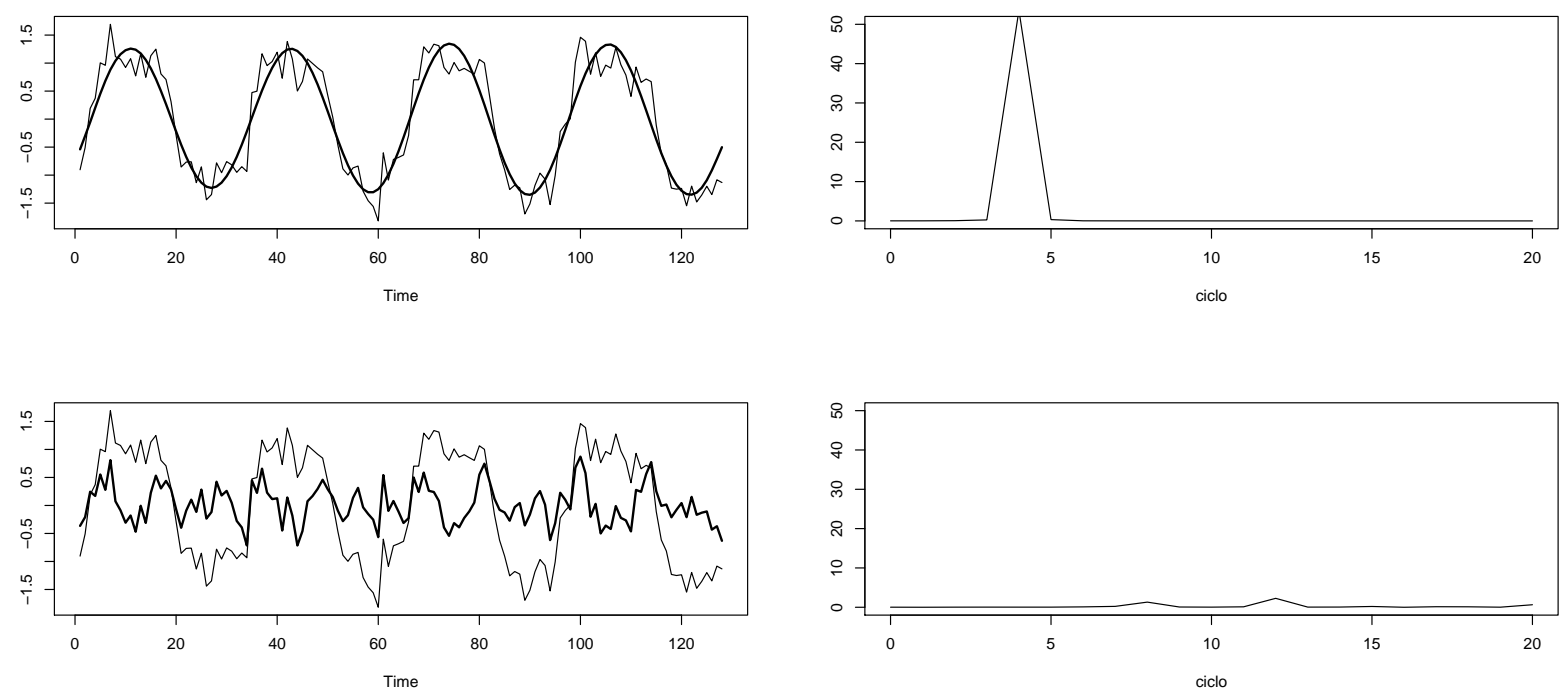

Figura 5.3: resultado para os grupos $\mathbf{H}^{(1)}$ e $\mathbf{H}^{(2)}$ 


\section{Capítulo 6}

\section{MSSA Multichannel Singular}

\section{Spectrum Analysis}

O método MSSA é uma generalização do SSA, em que o objetivo é identificar padrões como: tendência, oscilação, periodicidade e ruído de um conjunto de séries de tempo. Assim, estamos interessados em capturar estruturas que representem um comportamento mais abrangente e leve em consideração os efeitos entre um conjunto de $L$ séries de tempo. O método MSSA é muito aplicado para dados meteorológicos, por isso, grande parte das séries de tempo são geralmente representadas por localidades ou regiões de um mapa.

O método MSSA segue a mesma estrutura de análise do SSA contendo as mesmas etapas complementares de decomposição e reconstrução, com algumas particularidades na estrutura da matriz de covariância $\mathbf{S}$ e na escolha do tamanho da janela $M$.

Na decomposição, de forma idêntica à construção univariada SSA, construímos a matriz de trajetórias para cada uma das $L$ séries de tempo $X_{l t}, l=1,2, \ldots, L$.

Utilizando as informações da matriz de trajetórias das $L$ séries, calculamos a matriz de covariâncias $\mathbf{S}$ de dimensão $(M L \times M L)$.

A matriz $\mathbf{S}$ contém informação cruzada entre os lags de tempo para cada e entre cada uma das $L$ séries em sua estrutura. Na etapa de decomposição, construímos a matriz $\mathbf{S}$ e determinamos os autovalores e autovetores associados. Em seguida, na etapa de reconstrução, usamos as informações da decomposição para encontrarmos estruturas e características globais e relevantes para as $L$ séries de tempo. 
Comparando com SSA, notamos que a principal diferença consiste em que o resultado leva em consideração as estruturas cruzadas das $L$ séries de tempo. Particularmente, se $L=1$, temos o resultado para SSA, se $\mathrm{L}>1$ e $M=1$, temos o resultado da análise de componentes principais do caso clássico.

Mais detalhes sobre o método podem ser encontrados em Jolliffe [2002], Plaut e Vautard [1994], von Storch e Zwiers [1999], Guil et al. [2002].

\subsection{Escolha do Tamanho da Janela $M$ e da Quantidade de Grupos}

Para escolha do tamanho da janela $M$ e da quantidade de grupos para reconstrução das séries, os mesmos procedimentos sugeridos para análise SSA são recomendados.

Vimos na análise SSA que quando estávamos diante de uma estrutura complexa e com grande quantidade de observações, poderíamos escolher um valor de $M$ próximo a $N / 2$. No entanto para MSSA, o tamanho da janela $M$ e a quantidade de séries podem gerar um volume muito grande de informações, tornando inviável a análise.

Desta forma, conhecer a série de tempo, obtendo previamente características sobre ela, facilita a escolha do tamanho da janela para análise.

De forma geral, tudo que foi descrito anteriormente para escolha de $M$ e para reconstrução da quantidade de grupos no SSA será usado no método MSSA.

\subsection{Primeira Etapa: Decomposição}

Suponha $X_{l t}$ a série de tempo para a $l$-ésima localidade para $t$ unidades de tempo $t=1, \ldots, N$. Desta forma, construímos a matriz de trajetórias a partir de um tamanho de janela igual a $M$ obtido pela análise das informações disponíveis sobre as $L$ séries.

Temos para o estudo, observações de $L$ séries de tempo para $N$ unidades de tempo e construímos $L$ matrizes de trajetórias $\mathbf{H}_{l}, l=1, \ldots, L$, uma para cada série de tempo $X_{l t}$, todas com a mesma dimensão $(M \times(N-M+1))$.

Denotamos por $\mathbf{H}_{l}$ a matriz de trajetórias da série $\mathbf{X}_{l}=\left(X_{l 1}, X_{l 2}, \ldots, X_{l N}\right)^{\prime}$ para a localização, 
em que os elementos $h_{l t}$, são os elementos de $X_{l}$ no instante de tempo $t$, ou seja $X_{l t}$.

A seguir, mostramos a estrutura das matrizes de trajetórias. Para facilitar a descrição da etapa vamos adotar duas localidades, isto é $\mathrm{L}=2$. Assim temos as respectivas matrizes $\mathbf{H}_{1}$ e $\mathbf{H}_{2}$ para $l=1$ e $l=2$ :

$$
\begin{gathered}
\mathbf{H}_{1}=\left[\begin{array}{ccccc}
h_{11} & h_{12} & h_{13} & \cdots & h_{1 N-M+1} \\
h_{12} & h_{13} & h_{14} & \cdots & h_{1 N-M+2} \\
h_{13} & h_{14} & h_{15} & \cdots & h_{1 N-M+3} \\
\vdots & \vdots & \vdots & \ddots & \vdots \\
h_{1 M} & h_{1 M+1} & h_{1 M+2} & \cdots & h_{1 N}
\end{array}\right] \\
\mathbf{H}_{2}=\left[\begin{array}{ccccc}
h_{21} & h_{22} & h_{23} & \cdots & h_{2 N-M+1} \\
h_{22} & h_{23} & h_{24} & \cdots & h_{2 N-M+2} \\
h_{23} & h_{24} & h_{25} & \cdots & h_{2 N-M+3} \\
\vdots & \vdots & \vdots & \ddots & \vdots \\
h_{2 M} & h_{2 M+1} & h_{2 M+2} & \cdots & h_{2 N}
\end{array}\right]
\end{gathered}
$$

Neste caso, a matriz $\mathbf{H}$ será:

$$
\mathbf{H}=\left[\begin{array}{l}
\mathbf{H}_{1} \\
\mathbf{H}_{2}
\end{array}\right]
$$


$\mathrm{Ou}$

$$
\mathbf{H}=\left[\begin{array}{ccccc}
h_{11} & h_{12} & h_{13} & \cdots & h_{1 N-M+1} \\
h_{12} & h_{13} & h_{14} & \cdots & h_{1 N-M+2} \\
\vdots & \vdots & \vdots & \ddots & \vdots \\
h_{1 M} & h_{1 M+1} & h_{1 M+2} & \cdots & h_{1, N} \\
& & & & \\
h_{21} & h_{22} & h_{23} & \cdots & h_{2 N-M+1} \\
h_{22} & h_{23} & h_{24} & \cdots & h_{2 N-M+2} \\
\vdots & \vdots & \vdots & \ddots & \vdots \\
h_{2 M} & h_{2 M+1} & h_{2 M+2} & \cdots & h_{2 N}
\end{array}\right]
$$

Com isso, cada uma das localidades terá uma matriz de trajetórias $\mathbf{H}_{l}$ de tamanho $(M \times$ $(N-M+1))$. Calculamos agora a matriz de covariância $\mathbf{S}$ que terá em sua diagonal principal $\left(\mathbf{S}_{11}\right.$ a covariância entre as colunas da matriz $\left.\mathbf{H}_{1}\right),\left(\mathbf{S}_{22}\right.$ a covariância entre as colunas da matriz $\left.\mathbf{H}_{2}\right), \ldots,\left(\mathbf{S}_{L L}\right.$ a covariância entre as colunas da matriz $\left.\mathbf{H}_{L}\right)$, e fora da diagonal, $\mathbf{S}_{L k}$ para $l \neq k$, a covariância cruzada entre as colunas das matrizes $\mathbf{H}_{l}$ e $\mathbf{H}_{k}$.

Conforme mencionado, a matriz $\mathbf{S}$ terá dimensão $(M L \times M L)$ e os seus elementos $\mathbf{S}_{i j}$ terão estrutura tipo Toeplitz de dimensão $(M \times M)$.

Desta forma, a matriz $\mathbf{S}$ terá uma estrutura Block-Toeplitz em que os elementos da sua diagonal, $\mathbf{S}_{11}, \mathbf{S}_{22}, \ldots, \mathbf{S}_{L L}$ serão respectivamente as mesmas da análise SSA se estivéssemos analisando respectivamente $X_{1 t}, X_{2 t}, \ldots, X_{L t}$ individualmente.

A seguir descrevemos, como exemplo, a estrutura de uma matriz $\mathbf{S}$ com $L=2$ e $M=3$.

$$
\mathbf{S}=\left[\begin{array}{ll}
\mathbf{S}_{11} & \mathbf{S}_{12} \\
\mathbf{S}_{21} & \mathbf{S}_{22}
\end{array}\right]
$$

ou ainda $s_{i j}^{l k}$ o valor respectivo da covâriancia entre as localidades $l$ e $k$ e entre os lags da 
série $i$ e $j$.

$$
\mathbf{S}=\left[\begin{array}{lllllll}
s_{11}^{11} & s_{12}^{11} & s_{13}^{11} & & s_{11}^{12} & s_{12}^{12} & s_{13}^{12} \\
& & & & & \\
s_{21}^{11} & s_{22}^{11} & s_{23}^{11} & & s_{21}^{12} & s_{22}^{12} & s_{23}^{12} \\
s_{31}^{11} & s_{32}^{11} & s_{33}^{11} & & s_{31}^{12} & s_{32}^{12} & s_{33}^{12} \\
& & & & & & \\
s_{11}^{21} & s_{12}^{21} & s_{13}^{21} & & s_{11}^{22} & s_{12}^{22} & s_{13}^{22} \\
& & & & & \\
s_{21}^{21} & s_{22}^{21} & s_{23}^{21} & & s_{21}^{22} & s_{22}^{22} & s_{23}^{22} \\
& & & & & & \\
s_{31}^{21} & s_{32}^{21} & s_{33}^{21} & & s_{31}^{22} & s_{32}^{22} & s_{33}^{22}
\end{array}\right]
$$

Construída a matriz $\mathbf{S}$ conseguimos transformar as $L$ séries em componentes aditivas, através da decomposição do valor singular da matriz $\mathbf{H}$.

Assim, para $L=2$ e $M=3$, temos os respectivos autovalores e autovetores: $\lambda_{1}, \lambda_{2}, \ldots, \lambda_{6}$ e $\mathbf{U}_{1}$, $\mathbf{U}_{2}, \ldots, \mathbf{U}_{6}$ da matriz $\mathbf{S}$ e assumindo $d=\max \left[i\right.$, tal que $\left.\lambda_{i}>0\right]$, temos que as matrizes de trajetórias $\mathbf{H}_{1}$ e $\mathbf{H}_{2}$ podem ser escritas como as seguintes decomposições aditivas:

$$
\begin{aligned}
& \mathbf{H}_{1}=\mathbf{H}_{1, I_{1}}+\ldots+\mathbf{H}_{1, I_{d}} \\
& \mathbf{H}_{2}=\mathbf{H}_{2, I_{1}}+\ldots+\mathbf{H}_{2, I_{d}}
\end{aligned}
$$

Definimos $\mathbf{H}_{I_{i}}$ como a matriz que contém os elementos de $\mathbf{H}_{1, I_{i}}$ e $\mathbf{H}_{2, I_{i}}$, desta forma:

$$
\mathbf{H}_{I_{i}}=\left[\begin{array}{c}
\mathbf{H}_{1, I_{i}} \\
\mathbf{H}_{2, I_{i}}
\end{array}\right],
$$

em que $\mathbf{H}_{I_{i}}=\sqrt{\lambda}_{i} \mathbf{U}_{i} \mathbf{V}_{i}^{\prime}$ e $\mathbf{V}_{i}=\mathbf{X}^{\prime} \mathbf{U}_{i} / \sqrt{\lambda}_{i}(i=1, \ldots, d)$. Neste caso, as $M$ primeiras linhas da matriz $\mathbf{H}_{I_{i}}$ estão associadas a $\mathbf{H}_{1, I_{i}}$ e as últimas $M$ linhas associadas a $\mathbf{H}_{2, I_{i}}$, para 
$i=1, \ldots, d$.

Assim teremos a combinação do valor singular da matriz $\mathbf{H}$ com as respectivas combinações dos valores $\left(\sqrt{\lambda}_{i}, \mathbf{U}_{i}, \mathbf{V}_{i}\right)$, denominado autotriplo da decomposição.

Nota-se que é possível retornarmos a cada uma das $L$ séries originais, utilizando a soma de cada uma das respectivas partes/linhas de cada uma das matriz $\mathbf{H}_{I_{i}}$.

Verifica-se que o resultado dos autovalores e autovetores são extraídos da matriz de covariância das localidades e leva em consideração as covariâncias cruzadas entre as regiões, o que torna o resultado diferente do que simplesmente capturarmos a estrutura de cada uma das $L$ séries pelo método SSA.

\subsection{Segunda Etapa: Reconstrução}

Para a reconstrução, seguimos o mesmo procedimento univariado SSA, encontramos primeiro os grupos de decomposições: $\left(\mathbf{H}_{1}^{(1)}, \ldots, \mathbf{H}_{1}^{(r)}\right),\left(\mathbf{H}_{2}^{(1)}, \ldots, \mathbf{H}_{2}^{(r)}\right), \ldots,\left(\mathbf{H}_{L}^{(1)}, \ldots, \mathbf{H}_{L}^{(r)}\right)$ em que cada $\mathbf{H}_{l}^{(i)}$ representa uma característica ou estrutura para a localidade $l$ do conjunto das séries de tempo $X_{l t}$, em que $\mathbf{H}_{l}^{(i)}$ é construída agrupando os valores de $\mathbf{H}_{l, I_{i}}$ para cada uma das $L$ localidades.

Suponda que a decomposição gerou $d$ partes aditivas de $\mathbf{H}_{1}, \mathbf{H}_{2}, \ldots, \mathbf{H}_{L}$, tal que:

$$
\begin{gathered}
\mathbf{H}_{1}=\mathbf{H}_{1, I_{1}}+\ldots+\mathbf{H}_{1, I_{d}} \\
\mathbf{H}_{2}=\mathbf{H}_{2, I_{1}}+\ldots+\mathbf{H}_{2, I_{d}} \\
\vdots \\
\mathbf{H}_{L}=\mathbf{H}_{L, I_{1}}+\ldots+\mathbf{H}_{L, I_{d}}
\end{gathered}
$$

Como exemplo, podemos supor forte estrutura periódica configurada nas séries $X_{l t}$. Desta forma, devemos encontrar a periodicidade associada a cada localidade, por exemplo, nos elementos $\mathbf{H}_{1}^{(1)}$ para a localidade $1, \mathbf{H}_{2}^{(1)}$ para a localidade $2, \ldots, \mathbf{H}_{L}^{(1)}$ para a localidade $L$ e de forma análoga podemos encontrar as $r$ estruturas das séries. 
Assim, supondo a presença de 2 estruturas, $\mathrm{r}=2$, e 3 localidades, $L=3$, teremos as seguintes configurações para $\mathbf{H}_{l}^{(r)}$ :

$$
\begin{aligned}
\mathbf{H}_{1}^{(1)} & =\sum_{i=1}^{d_{1}} \mathbf{H}_{1, I_{i}} \\
\mathbf{H}_{1}^{(2)} & =\sum_{i=d_{1}+1}^{d} \mathbf{H}_{1, I_{i}} \\
\mathbf{H}_{2}^{(1)} & =\sum_{i=1}^{d_{1}} \mathbf{H}_{2, I_{i}} \\
\mathbf{H}_{2}^{(2)} & =\sum_{i=d_{1}+1}^{d} \mathbf{H}_{2, I_{i}} \\
\mathbf{H}_{3}^{(1)} & =\sum_{i=1}^{d_{1}} \mathbf{H}_{3, I_{i}} \\
\mathbf{H}_{3}^{(2)} & =\sum_{i=d_{1}+1}^{d} \mathbf{H}_{3, I_{i}}
\end{aligned}
$$

Nota-se uma estrutura ampliada do modelo SSA, e segue assim que:

$$
\begin{aligned}
& \mathbf{H}_{1}=\mathbf{H}_{1}^{(1)}+\mathbf{H}_{1}^{(2)} \\
& \mathbf{H}_{2}=\mathbf{H}_{2}^{(1)}+\mathbf{H}_{2}^{(2)} \\
& \mathbf{H}_{3}=\mathbf{H}_{3}^{(1)}+\mathbf{H}_{3}^{(2)}
\end{aligned}
$$

Deseja-se que a separação seja de tal forma que cada uma das matrizes $\mathbf{H}_{1}^{(1)}, \mathbf{H}_{1}^{(2)}, \mathbf{H}_{2}^{(1)}$, $\mathbf{H}_{2}^{(2)}, \mathbf{H}_{3}^{(1)}$ e $\mathbf{H}_{3}^{(2)}$ tenha uma estrutura próxima de Hankel e cada uma seja transformada, por diagonalização, em um vetor com $N$ elementos. 
Portanto, teremos respectivamente para cada $\mathbf{H}_{l}^{(i)}$ o seguinte resultado:

$$
\mathbf{H}_{l}^{(i)}=\left[\begin{array}{ccccc}
h_{l, 1,1}^{(i)} & h_{l, 1,2}^{(i)} & h_{l, 1,3}^{(i)} & \cdots & h_{l, 1, K}^{(i)} \\
h_{l, 2,1}^{(i)} & h_{l, 2,3}^{(i)} & h_{l, 2,4}^{(i)} & \cdots & h_{l, 2, K}^{(i)} \\
h_{l, 3,1}^{(i)} & h_{l, 3,2}^{(i)} & h_{l, 3,3}^{(i)} & \cdots & h_{l, 3, K}^{(i)} \\
\vdots & \vdots & \vdots & \ddots & \vdots \\
h_{l, M, 1}^{(i)} & h_{l, M, 2}^{(i)} & h_{l, M, 3}^{(i)} & \cdots & h_{l, M, K}^{(i)}
\end{array}\right]
$$

em que $K=N-M+1$.

e para $\widetilde{\mathbf{H}_{l}^{(i)}}$, teremos:

$$
\widetilde{\mathbf{H}_{l}^{(i)}}=\left[\begin{array}{c}
h_{l, 1,1}^{i} \\
\frac{h_{l, 1,2}^{i}+h_{l, 2,1}^{i}}{2} \\
\frac{h_{l, 1,3}^{i}+h_{l, 2,3}^{i}+h_{l, 3,1}^{i}}{3} \\
\vdots \\
\frac{h_{l, M-2, K}^{i}+h_{l, M-1, K-1}^{i}+h_{l, M, K-2}^{i}}{3} \\
\frac{h_{l, M-1, K}^{i}+h_{l, M, K-1}^{i}}{2} \\
h_{l, M, K}^{(i)}
\end{array}\right]
$$

em que $\widetilde{\mathbf{H}_{l}^{(i)}}$ tem dimensão $(N \times 1)$, para cada $i$ representando uma estrutura para a série de tempo da localidade $l$, como tendência, periodicidade ou ruído da série.

Portanto, $\widetilde{\mathbf{H}_{l}^{(1)}}$ e $\widetilde{\mathbf{H}_{l}^{(2)}}$ representarão as mesmas características associadas às $L$ séries, por exemplo, a primeira delas pode representar a estrutura periódica das séries e a segunda uma outra característica. 


\subsection{Aplicação de MSSA para Dados fMRI}

Neste exemplo, utilizamos os mesmos dados em que aplicamos os exemplos da análise de componentes principais pelo método clássico e a análise pelo domínio da frequência.

Para o estudo, contamos com 8 variáveis $\mathbf{X}_{t}=\left(X_{1 t}, X_{2 t}, X_{3 t}, X_{4 t}, X_{5 t}, X_{6 t}, X_{7 t}, X_{8 t}\right)$ que representam medidas consecutivas do nível de oxigenação do sangue segundo as $N$ unidades de tempo.

Para MSSA, iniciamos a análise identificando o tamanho da janela $M$, que determinará a quantidade de componentes principais que iremos analisar.

Conforme apresentado na Figura 2.1 e nos periodogramas da Figura 4.1, observamos que grande parte das 8 séries apresentam forte estrutura periódica com período $T$ igual a 128/4=32 segundos, causado pela amostra a cada 2 segundos de estímulos dado a cada 32 segundos e interrompido por 32 segundos durante 256 segundos. A escolha do tamanho da janela, seguindo o que foi apresentado anteriormente no item de escolha do tamanho da janela para dados periódicos, será dada por $M / T=1$, ou seja $M=32$.

A escolha de $M=32$, implica que teremos para cada uma das 8 séries, uma matriz de trajetórias de dimensão $(M \times(N-M+1))$ ou $(32 \times 97)$, conforme abaixo:

$$
\mathbf{H}_{l}=\left[\begin{array}{ccccc}
h_{l, 1} & h_{l, 2} & h_{l, 3} & \cdots & h_{l, 97} \\
h_{l, 2} & h_{l, 3} & h_{l, 4} & \cdots & h_{l, 98} \\
h_{l, 3} & h_{l, 4} & h_{l, 5} & \cdots & h_{l, 99} \\
\vdots & \vdots & \vdots & \ddots & \vdots \\
h_{l, 32} & h_{l, 33} & h_{l, 34} & \cdots & h_{l, 128}
\end{array}\right], l=1, \ldots, 8 .
$$

Neste caso, a matriz $\mathbf{H}$ será dada por:

$$
\mathbf{H}=\left[\begin{array}{c}
\mathbf{H}_{1} \\
\vdots \\
\mathbf{H}_{8}
\end{array}\right]
$$


$\mathrm{Ou}$

$$
\mathbf{H}=\left[\begin{array}{ccccc}
h_{1,1} & h_{1,2} & h_{1,3} & \cdots & h_{1,97} \\
h_{1,2} & h_{1,3} & h_{1,4} & \cdots & h_{1,98} \\
\vdots & \vdots & \vdots & \ddots & \vdots \\
h_{1,32} & h_{1,33} & h_{1,34} & \cdots & h_{1,128} \\
& & & & \\
h_{2,1} & h_{2,2} & h_{2,3} & \cdots & h_{2,97} \\
h_{2,2} & h_{2,3} & h_{2,4} & \cdots & h_{2,98} \\
\vdots & \vdots & \vdots & \ddots & \vdots \\
h_{2,32} & h_{2,33} & h_{2,34} & \cdots & h_{2,128} \\
& & & & \\
\vdots & \vdots & \vdots & \vdots & \vdots \\
& & & & \\
h_{8,1} & h_{8,2} & h_{8,3} & \cdots & h_{8,97} \\
h_{8,2} & h_{8,3} & h_{8,4} & \cdots & h_{8,98} \\
\vdots & \vdots & \vdots & \ddots & \vdots \\
h_{8,32} & h_{8,33} & h_{8,34} & \cdots & h_{8,128}
\end{array}\right]
$$

Partindo da matriz $\mathbf{H}$, construímos a matriz de covariâncias $\mathbf{S}$ da seguinte forma:

$$
\mathbf{S}=\left[\begin{array}{cccccccc}
\mathbf{S}_{11} & \mathbf{S}_{12} & \mathbf{S}_{13} & \mathbf{S}_{14} & \mathbf{S}_{15} & \mathbf{S}_{16} & \mathbf{S}_{17} & \mathbf{S}_{18} \\
\mathbf{S}_{21} & \mathbf{S}_{22} & \mathbf{S}_{23} & \mathbf{S}_{24} & \mathbf{S}_{25} & \mathbf{S}_{26} & \mathbf{S}_{27} & \mathbf{S}_{28} \\
\mathbf{S}_{31} & \mathbf{S}_{32} & \mathbf{S}_{33} & \mathbf{S}_{34} & \mathbf{S}_{35} & \mathbf{S}_{36} & \mathbf{S}_{37} & \mathbf{S}_{38} \\
\mathbf{S}_{41} & \mathbf{S}_{42} & \mathbf{S}_{43} & \mathbf{S}_{44} & \mathbf{S}_{45} & \mathbf{S}_{46} & \mathbf{S}_{47} & \mathbf{S}_{48} \\
\mathbf{S}_{51} & \mathbf{S}_{52} & \mathbf{S}_{53} & \mathbf{S}_{54} & \mathbf{S}_{55} & \mathbf{S}_{56} & \mathbf{S}_{57} & \mathbf{S}_{58} \\
\mathbf{S}_{61} & \mathbf{S}_{62} & \mathbf{S}_{63} & \mathbf{S}_{64} & \mathbf{S}_{65} & \mathbf{S}_{66} & \mathbf{S}_{67} & \mathbf{S}_{68} \\
\mathbf{S}_{71} & \mathbf{S}_{72} & \mathbf{S}_{73} & \mathbf{S}_{74} & \mathbf{S}_{75} & \mathbf{S}_{76} & \mathbf{S}_{77} & \mathbf{S}_{78} \\
\mathbf{S}_{81} & \mathbf{S}_{82} & \mathbf{S}_{83} & \mathbf{S}_{84} & \mathbf{S}_{85} & \mathbf{S}_{86} & \mathbf{S}_{87} & \mathbf{S}_{88}
\end{array}\right]
$$


em que $\mathbf{S}_{l l}$ é a matriz de covariância determinada pela covariância entre as colunas da matriz $\mathbf{H}_{l}$ e terá dimensão $(32 \times 32)$. Já $\mathbf{S}_{l k}=\mathbf{S}_{k l}^{\prime}$, é a matriz de covariâncias cruzadas entre as colunas das matrizes $\mathbf{H}_{l}$ e $\mathbf{H}_{k}$, para $l, k=1, \ldots, 8$. Assim, $\mathbf{S}$ terá dimensão $(M L \times M L)$, neste caso $(256 \times 256)$.

Com a matriz $\mathbf{S}$, calculamos os $d$ autovetores e autovalores da matriz $\mathbf{S}, d=\max [i$, tal que $\left.\lambda_{i}>0\right]$, respectivamente $\mathbf{U}_{1}, \ldots, \mathbf{U}_{d}$ e $\lambda_{1}, \ldots, \lambda_{d}$. Para este exemplo, encontramos 96 autovalores positivos, ou seja, $d=96$.

Assim, podemos escrever as matrizes $\mathbf{H}_{1}, \mathbf{H}_{2}, \ldots, \mathbf{H}_{8}$ em soma de componentes aditivas determinando a matriz $\mathbf{H}_{I_{i}}$, em que $\mathbf{H}_{I_{i}}=\sqrt{\lambda}_{i} \mathbf{U}_{i} \mathbf{V}_{i}^{\prime}$, e $\mathbf{V}_{i}=\mathbf{X}^{\prime} \mathbf{U}_{i} / \sqrt{\lambda}_{i}(i=1, \ldots, d)$, lembrando que $\lambda_{i}$ e $\mathbf{U}_{i}$ são repectivamente o $i$-ésimo autovalor e autovetor associados à $i$-ésima componente, para $i=1, \ldots, 96$.

A matriz $\mathbf{H}_{I_{i}}$, terá os seguintes elementos:

$$
\mathbf{H}_{I_{i}}=\left[\begin{array}{c}
\mathbf{H}_{1, I_{i}} \\
\mathbf{H}_{2, I_{i}} \\
\mathbf{H}_{3, I_{i}} \\
\mathbf{H}_{4, I_{i}} \\
\mathbf{H}_{5, I_{i}} \\
\mathbf{H}_{6, I_{i}} \\
\mathbf{H}_{7, I_{i}} \\
\mathbf{H}_{8, I_{i}}
\end{array}\right]
$$

Neste caso, as 32 primeiras linhas da matriz $\mathbf{H}_{I_{i}}$ estão associadas a $\mathbf{H}_{1, I_{i}}$ e as últimas 32 linhas associadas a $\mathbf{H}_{8, I_{i}}$, para $i=1, \ldots, 96$.

As matrizes $\mathbf{H}_{1, I_{i}}, \mathbf{H}_{2, I_{i}}, \ldots, \mathbf{H}_{8, I_{i}}$ terão dimensão $(M \times(N-M+1))$, ou seja $(32 \times 97)$, para todo $i=1, \ldots, 96$. Desta forma, podemos escrever $\mathbf{H}_{1}, \ldots, \mathbf{H}_{8}$, como:

$$
\begin{aligned}
& \mathbf{H}_{1}=\mathbf{H}_{1, I_{1}}+\ldots+\mathbf{H}_{1, I_{d}} \\
& \mathbf{H}_{2}=\mathbf{H}_{2, I_{1}}+\ldots+\mathbf{H}_{2, I_{d}}
\end{aligned}
$$




$$
\mathbf{H}_{8}=\mathbf{H}_{8, I_{1}}+\ldots+\mathbf{H}_{8, I_{d}}
$$

Verificamos que a maior contribuição para explicar as estruturas das séries estão nas 4 primeiras componentes, capturando a estrutura periódica dos dados e contribuindo representativamente com a variabilidade dos dados $\frac{\sum_{i=1}^{4} \lambda_{i}}{\sum_{i=1}^{96} \lambda_{i}}=0,6$.

Desta forma, separamos os efeitos dos dados observados $\mathbf{H}_{t}$ em uma estrutura periódica e outra estrutura não periódica. Assim chamaremos $\mathbf{H}_{l}^{(1)}$ a estrutura periódica para $l=1, \ldots, 8$ e $\mathbf{H}_{l}^{(2)}$ a estrutura não periódica para $l=1, \ldots, 8$.

Assim, para a estrutura periódica temos:

$$
\begin{gathered}
\mathbf{H}_{1}^{(1)}=\mathbf{H}_{1, I_{1}}+\mathbf{H}_{1, I_{2}}+\mathbf{H}_{1, I_{3}}+\mathbf{H}_{1, I_{4}} \\
\mathbf{H}_{2}^{(1)}=\mathbf{H}_{2, I_{1}}+\mathbf{H}_{2, I_{2}}+\mathbf{H}_{2, I_{3}}+\mathbf{H}_{2, I_{4}} \\
\vdots \\
\mathbf{H}_{8}^{(1)}=\mathbf{H}_{8, I_{1}}+\mathbf{H}_{8, I_{2}}+\mathbf{H}_{8, I_{3}}+\mathbf{H}_{8, I_{4}}
\end{gathered}
$$

e para a estrutura não periódica temos:

$$
\begin{gathered}
\mathbf{H}_{1}^{(2)}=\mathbf{H}_{1, I_{5}}+\ldots+\mathbf{H}_{1, I_{96}} \\
\mathbf{H}_{2}^{(2)}=\mathbf{H}_{2, I_{5}}+\ldots+\mathbf{H}_{2, I_{96}} \\
\vdots \\
\mathbf{H}_{8}^{(2)}=\mathbf{H}_{8, I_{5}}+\ldots+\mathbf{H}_{8, I_{96}}
\end{gathered}
$$

Seguem abaixo os primeiros elementos da matriz $\mathbf{H}_{1}^{(1)}$ e os primeiros elementos da matriz $\mathbf{H}_{2}^{(1)}$. Teremos um bom resultado quando as matrizes $\mathbf{H}_{l}^{(i)}, l=1, \ldots, 8$ e $i=1,2$, tiverem 
estruturas próximas de Hankel, ou seja, com elementos $h_{l, k}=h_{l-1, k+1}$.

$$
\begin{aligned}
\mathbf{H}_{1}^{(1)}= & {\left[\begin{array}{rrrrr}
-0,64 & -0,34 & -0,03 & 0,30 & \cdots \\
-0,40 & -0,05 & 0,28 & 0,58 & \cdots \\
-0,07 & 0,28 & 0,58 & 0,82 & \cdots \\
0,28 & 0,60 & 0,83 & 0.98 & \cdots \\
0,61 & 0,87 & 1,02 & 1,08 & \cdots
\end{array}\right] } \\
\mathbf{H}_{2}^{(1)}= & {\left[\begin{array}{rrrrrr}
-0,85 & -0,61 & -0,33 & -0,06 & \cdots \\
-0,67 & -0,37 & -0,08 & 0,18 & \cdots \\
-0,38 & -0,07 & 0,19 & 0,40 & \cdots \\
-0,11 & 0,18 & 0,40 & 0,55 & \cdots \\
0,15 & 0,41 & 0,58 & 0.68 & \cdots
\end{array}\right] . }
\end{aligned}
$$

Na etapa seguinte faremos a diagonalização das matrizes $\mathbf{H}_{l}^{(i)}, l=1, \ldots, 8$ e $i=1,2$, de forma que transformaremos as matrizes de tamanho $(M \times(N-M+1))$ em um vetor de tamanho $N$. Denotaremos respectivamente cada uma das diagonalizações por $\widetilde{\mathbf{H}_{l}^{(i)}}, l=1, \ldots, 8 \mathrm{e}$ $i=1,2$. Seguem abaixo as diagonalizaçãos das matrizes, $\mathbf{H}_{1}^{(1)}$ e $\mathbf{H}_{2}^{(1)}$.

$$
\widetilde{\mathbf{H}_{1}^{(1)}}=\left[\begin{array}{c}
-0,64 \\
\frac{-0,34-0,40}{2} \\
\frac{-0,03-0,05-0.07}{3} \\
\frac{0,30+0,28+0,28+0,28}{4} \\
\vdots \\
-0,47
\end{array}\right]
$$




$$
\widetilde{\mathbf{H}_{2}^{(1)}}=\left[\begin{array}{c}
-0,85 \\
\frac{-0,61-0,67}{2} \\
\frac{-0,33-0,37-0,38}{3} \\
\frac{-0,06-0,08-0,07-0.11}{4} \\
\vdots \\
-0,69
\end{array}\right]
$$

Na Figura 6.1, mostramos os gráficos das séries originais $X_{1 t}, X_{2 t}, \ldots, X_{8 t}$ e comparamos com os resultados de $\widetilde{\mathbf{H}_{l}^{(1)}}, l=1, \ldots, 8$. A linha contínua representa os dados observados e a linha pontilhada mostra o efeito periódico ajustado pelo método.

Na Figura 6.2, mostramos os gráficos das séries originais $X_{1 t}, X_{2 t}, \ldots, X_{8 t}$ e comparamos com os resultados de $\widetilde{\mathbf{H}_{l}^{(2)}}, l=1, \ldots, 8$. A linha contínua representa os dados observados e a linha pontilhada mostra o efeito não periódico ajustado pelo método.

Os resultados apresentados nas Figuras 6.1 e 6.2 mostram que foi possível encontrar de forma satisfatória uma estrutura periódica causada pela resposta ao estímulo de cada uma das 8 variáveis. Mostramos que além de identificarmos, conseguimos separá-las em $\mathbf{H}_{l}^{(1)}$ e $\mathbf{H}_{l}^{(2)}, l=1, \ldots, 8$.

Podemos constatar satisfatoriamente a separação pela proximidade de uma matriz de Hankel, $\mathbf{H}_{l}^{(1)}$ e $\mathbf{H}_{l}^{(1)}$, mostrando assim que é possível separar $\mathbf{X}_{t}$ em um efeito periódico e outro não periódico conforme apresentado. 


\subsection{Sobre os Dados e Resultados}

A análise tem como objetivo capturar o comportamento ou estrutura de algum fenômeno, muitas vezes, o comportamento periódico que foi encontrado pela soma dos dois maiores autovalores, como no exemplo acima.

A análise apresentada poderia ser aplicada ao comportamento da temperatura do mar medida em duas posições, ou a medida de algum fenômeno em duas localidades. 

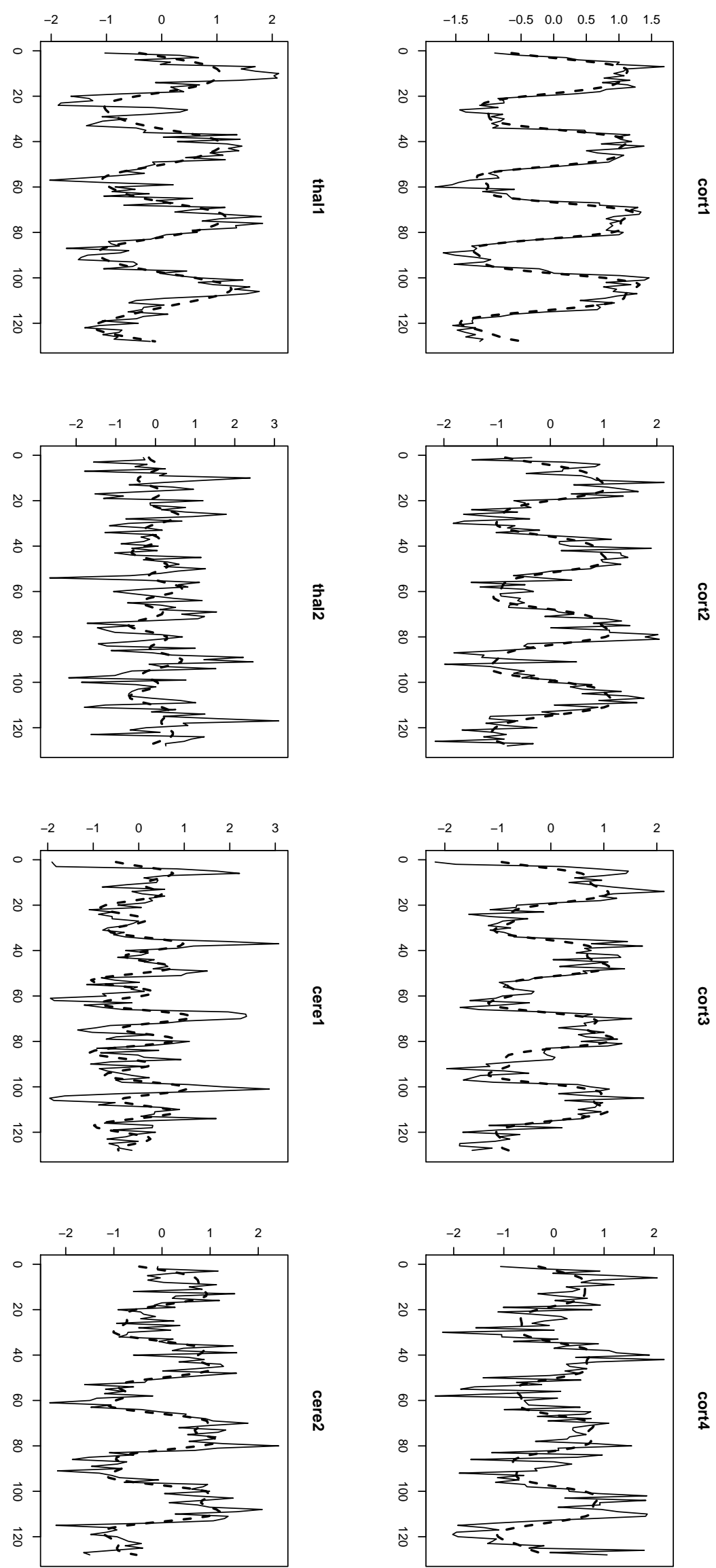

Figura 6.1: Séries observadas fMRI (linha contínua) e séries dos efeitos periódicos encontrados pelo método (linha pontilhada). 

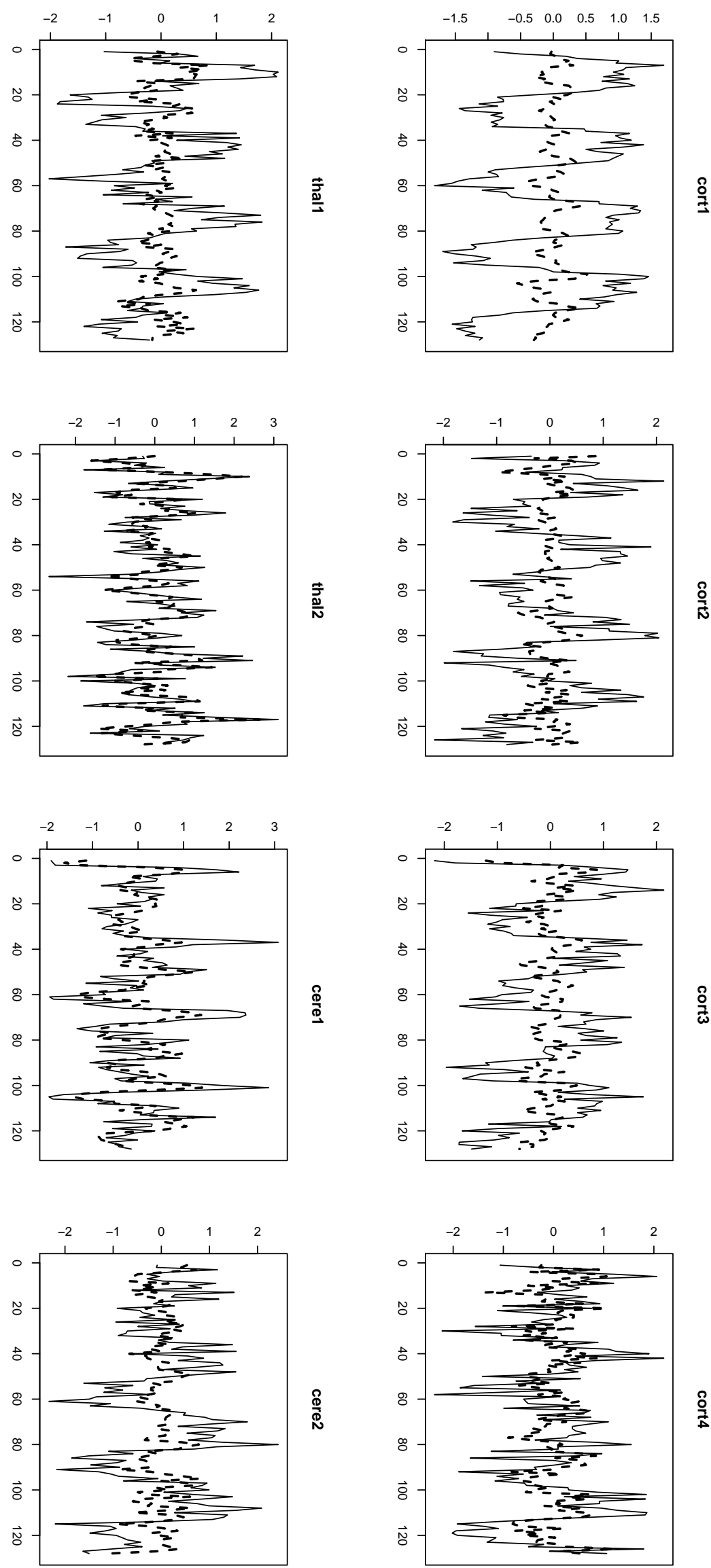

Figura 6.2: Séries observadas fMRI (linha contínua) e séries dos efeitos não periódicos encontrados pelo método (linha pontilhada). 


\section{Capítulo 7}

\section{Considerações Finais}

\subsection{Conclusões}

Em situações práticas o método de análise de componentes principais clássico é muito útil. A aplicação do método em séries temporais ignora a existência de dados correlacionados sequencialmente no tempo e por consequência, em algumas situações, o resultado encontrado pode deixar de capturar importantes informações, uma vez que a matriz de covariância é insuficiente para capturar a estrutura de autocorrelação dos dados observados.

Mostramos que a análise de componentes no domínio da frequência possibilita a análise de componentes principais para diferentes frequências $\lambda$. Desta forma, um conjunto de variáveis com o mesmo ciclo de tempo, como mostramos no exemplo dos dados fMRI, apresenta pouca diferença em comparação com o método clássico. No exemplo isso é devido à coleta da mesma resposta ao estímulo nas diferentes localidades do cérebro. Por outro lado, o método possibilida a análise para séries com diferentes ciclos de tempo, permitindo determinar e comparar diferentes componentes principais para diferentes valores de $\lambda$.

No método do domínio da frequência podemos, por meio das componentes, indentificar importantes características sobre os dados que não são observados no método clássico e/ou estão misturadas nas suas componentes.

Nos métodos SSA (Singular Spectrum Analysis) e MSSA (Multichannel Singular Spectrum Analysis), trabalhamos com os dados observados no domínio do tempo. Nesses métodos a análise é feita com base na relação entre as observações em diferentes unidades de tempo e 
as diferentes variáveis observadas, e são muito utilizados para dados atmosféricos na identificação de padrões, tais como: tendência e periodicidade.

Neste trabalho, mostramos na aplicação do método SSA que conseguimos separar duas características importantes. A primeira, correspondente à estrutura periódica da série, associada à resposta ao estímulo aplicado a cada 32 segundos, e a segunda, correspondente à estrutura não periódica. Mostramos pelo periodograma das duas características que, pelo método, conseguimos separar satisfatoriamente o efeito periódico dado pelo estímulo.

Vimos que o método MSSA é o caso multivariado do SSA e será um caso particular de PCA quando o tamanho da janela for $M=1$.

$\mathrm{Na}$ análise, assim como no caso SSA, conseguimos encontrar as mesmas estruturas: periódica e não periódica dos dados fMRI, em que os padrões ou estruturas identificadas leva em consideração a estrutura temporal entre as $L>1$ diferentes variáveis.

De forma simplificada, os métodos apresentados são alternativas à análise de componentes principais caso clássico. Pelo domínio da frequência podemos encontrar as componentes principais segundo os maiores espectros de cada uma das séries observadas tornando a análise mais específica. Nos métodos SSA e MSSA, identificamos as principais estruturas reponsáveis pela estrutura de correlação e autocorrelação das variáveis.

\subsection{Sugestões para Pesquisas Futuras}

No desenvolvimento deste trabalho, exibimos técnicas interessantes utilizadas para detecção de estruturas oscilatórias aplicadas geralmente a dados atmosféricos, mas que poderiam ser úteis para outras áreas.

Poderíamos elaborar um trabalho, não exclusivo à análise de componentes principais, dedicado à identificação de estruturas oscilatórias em dados atmosféricos.

A análise pelo método MSSA impõe que todas as séries sejam agrupadas em $r$ grupos. Uma pesquisa futura poderia ser dedicada a uma generalização do método que permita número diferentes de grupos para as séries consideradas. 


\section{Referências Bibliográficas}

Brillinger(2001) David R. Brillinger. Time Series: Data Analysis and Theory. SIAM: Society for Industrial and Applied Mathematics. Citado na pág. 21

Cai e Baines(2001) Wenju Cai e Peter G. Baines. Forcing of the antarctic circumpolar wave by el-nino-southern oscillation teleconnections. Journal of Geophysical Research, 106 (C5):9019-9038. Citado na pág. 2

Fisher(1929) R. A. Fisher. Tests of significance in harmonic analysis. Proceeding of the Royal Society, Series A, 125:54-59. Citado na pág. 25

Golyandina et al.(2001) Nina Golyandina, Vladimir Nekrutkin e Anatoly Zhigljavsky. Analysis of Time Series Struture SSA and Related Techniques. Chapman and Hall/CRC, primeira edição. Citado na pág. 31, 33, 37, 38, 39

Guil et al.(2002) M. Guil, K. Ide D. Kondrashov M. E Mann A. W. Robertson A. Saunders Y. Tian F. Varadi M.R Allen, M.D Dettinger e P.Yiou. Advanced spectral methods for climatic time series. Reviews of Geophysics. Citado na pág. 46

Hotelling(1933) Harold Hotelling. Analysis of a complex of statistical variable into principal component. J. Educational Psychol, 24:417-441; 498-520. Citado na pág. 1, 5

Johnson e Wichern(1998) Richard A. Johnson e Dean W. Wichern. Applied Multivariate Statistical Analysis. Prentice-Hall, 4th edição. Citado na pág. 6

Jolliffe(2002) I.T. Jolliffe. Principal Component Analysis. Springer, segunda edição. Citado na pág. $21,31,46$

Kim e Wu(1998) Kwang-Y. Kim e Qigang Wu. A comparison study of eof techniques: Analysis of nonstationary data with periodic statistics. Journal of Climate, páginas 185199. Citado na pág. 2

Lagerlund et al.(2004) Terrence D. Lagerlund, Frank W. Sharbrough e Neil E. Busacker. Use of principal component analysis in the frequency domain for mapping electroencephalografic activies: Comparison with phase-encoded fourier spectral analysis. Brain Topography, 17(2):73-84. Citado na pág. 21, 28, 29

Lorenz(1956) E.N. Lorenz. Empirical orthogonal functions and statistical weather prediction. Relatório Técnico MIT (NTIS AD 110268), Science Report 1, Statistical Forecasting Project, Department of Meteorology, 49pp. Citado na pág. 2

McDougall et al.(1995) A.J. McDougall, D.S. Stoffer e D.E. Tyler. Optimal transformations and the spectral envelope for real-valued time series. Journal of Stastistical Planning and Inference, 57:195-214. Citado na pág. 21 
Morettin(1999) Pedro A. Morettin. Ondas e Ondaletas, da análise de Fourier à análise de ondaletas. Edusp. Citado na pág. 11

Morettin e Toloi(2006) Pedro A. Morettin e Clélia M.C. Toloi. Análise de séries temporais. Egard Blucher, segunda edição. Citado na pág. 11, 25

Obukhov(1947) A.M. Obukhov. Statistically homogeneous fields on a sphere. Uspethi Mathematicheskikh Nauk, 2:196-198. Citado na pág. 2

Pearson(1901) Karl Pearson. On lines and planes of closest fit to systems of points in space. Philosophical Magazine, 2:559-572. Citado na pág. 1, 5

Plaut e Vautard(1994) Guy Plaut e Robert Vautard. Spell of low-frequency oscillations and weather regimes in the northern hemisphere. Journal of the Atmospheric Sciences, 51(2):210-236. Citado na pág. 46

Rencher(1997) Alvin C. Rencher. Multivariate Stastistical Inference and Applications. Wiley-Interscience, primeira edição. Citado na pág. 6

Shumway e Stoffer(2006) Robert H. Shumway e David S. Stoffer. Time Series Analysis and its Applications With $R$ Examples. Springer, segunda edição. Citado na pág. 6, 21

von Storch e Zwiers(2002) Hans von Storch e Francis W. Zwiers. Statistical Analysis in Climate Research. Cambridge University Press. Citado na pág. 2

von Storch e Zwiers(1999) Hans von Storch e Francis W. Zwiers. Analysis of Climate Variability: Applications of Statistical Techniques. Springer. Citado na pág. 46

Wilks(2011) Daniel S. Wilks. Statistical Methods in the Atmospheric Sciences. Academic Press, terceira edição. Citado na pág. 1, 31 